\title{
A Survey of Recent Trends in Multiobjective Optimal Control-Surrogate Models, Feedback Control and Objective Reduction
}

\author{
Sebastian Peitz * (D) and Michael Dellnitz \\ Chair of Applied Mathematics, Faculty for Computer Science, Electrical Engineering and Mathematics, \\ Paderborn University, Warburger Str. 100, 33098 Paderborn, Germany; dellnitz@uni-paderborn.de \\ * Correspondence: speitz@math.upb.de
}

Received: 15 May 2018; Accepted: 31 May 2018; Published: 1 June 2018

\begin{abstract}
Multiobjective optimization plays an increasingly important role in modern applications, where several criteria are often of equal importance. The task in multiobjective optimization and multiobjective optimal control is therefore to compute the set of optimal compromises (the Pareto set) between the conflicting objectives. The advances in algorithms and the increasing interest in Pareto-optimal solutions have led to a wide range of new applications related to optimal and feedback control, which results in new challenges such as expensive models or real-time applicability. Since the Pareto set generally consists of an infinite number of solutions, the computational effort can quickly become challenging, which is particularly problematic when the objectives are costly to evaluate or when a solution has to be presented very quickly. This article gives an overview of recent developments in accelerating multiobjective optimal control for complex problems where either PDE constraints are present or where a feedback behavior has to be achieved. In the first case, surrogate models yield significant speed-ups. Besides classical meta-modeling techniques for multiobjective optimization, a promising alternative for control problems is to introduce a surrogate model for the system dynamics. In the case of real-time requirements, various promising model predictive control approaches have been proposed, using either fast online solvers or offline-online decomposition. We also briefly comment on dimension reduction in many-objective optimization problems as another technique for reducing the numerical effort.
\end{abstract}

Keywords: multiobjective optimization; optimal control; model order reduction; model predictive control

MSC: 90C29; 49M37

\section{Introduction}

There is hardly ever a situation where only one goal is of interest at the same time. When performing a purchase for example, we want to pay a low price while getting a high quality product. In the same manner, multiple goals are present in most technical applications, maximizing quality versus minimizing the cost being only one of many examples. This dilemma leads to the field of multiobjective optimization, where we want to optimize all relevant objectives simultaneously. However, this is obviously impossible as the above example illustrates. Generically, the different objectives contradict each other such that we are forced to choose a compromise. While we are usually satisfied with one optimal solution in the scalar-valued setting, there exists in general an infinite number of optimal compromises in the situation where multiple objectives are present. The set of these compromise solutions is called the Pareto set, the corresponding points in the objective space form the Pareto front. 
Since the solution to a Multiobjective Optimization Problem (MOP) is a set, it is significantly more expensive to compute than the optimum of a single objective problem, and many researchers devote their work to the development of algorithms for the efficient numerical approximation of Pareto sets. These advances have opened up new challenging application areas for multiobjective optimization. In optimal control, the optimization variable is not finite-dimensional, but rather a function, typically depending on time. The goal is to steer a dynamical system in such a way that one (or multiple) objective is minimized. Two particularly challenging control problems are feedback control and control problems constrained by Partial Differential Equations (PDEs). In the first case, the time for computing the Pareto set is strictly limited, often to a small fraction of a second. In the latter case, even the solution of single objective problems is often extremely time consuming so that the development of new algorithmic ideas is necessary to make these problems computationally feasible.

In fact, in situations like these, surrogate models or dimension reduction techniques are a promising approach for significantly reducing the computational effort and thereby enabling real-time applicability. This article gives an overview of recent advances in surrogate modeling for multiobjective optimal control problems, where the approach is to replace the underlying system dynamics by a reduced order model, which can be solved much faster. On the other hand, it introduces an approximation error, which has to be taken into account when analyzing convergence properties. The article is structured as follows. In Section 2, we are going to review some basics about multiobjective optimization, including the most popular solution methods. The two main challenges are addressed individually in the next sections, starting with expensive models in Section 3. There are also surrogate modeling approaches for MOPs that directly provide a mapping from the control variable to the objective function values. Since there already exist extensive surveys for this case (cf. [1-3], for instance), these are summarized only very briefly. In Section 4, real-time feedback control is discussed. Finally, we briefly discuss the question of dimension reduction in the number of objectives in Section 5 before concluding with a summary of further research directions in Section 6.

\section{Multiobjective Optimization}

In this section, the concepts of multiobjective optimization and Pareto optimality will be introduced and some widely-used solution methods will be summarized. More detailed introductions to multiobjective optimization can be found in, e.g., [4,5].

\subsection{Theory}

In multiobjective optimization, we want to minimize multiple objectives at the same time. Consequently, the fundamental difference from scalar optimization is that the objective function $J: \mathcal{U} \rightarrow \mathbb{R}^{k}$ is vector-valued. Hence, the general problem is of the form:

$$
\begin{array}{ll}
\min _{u \in \mathcal{U}} J(u)=\min _{u \in \mathcal{U}}\left(\begin{array}{c}
J_{1}(u) \\
\vdots \\
J_{k}(u)
\end{array}\right) \\
\text { s.t. } \quad g_{i}(u) \leq 0, \quad i=1, \ldots, l, \\
& h_{j}(u)=0, \quad j=1, \ldots, m,
\end{array}
$$

where $u \in \mathcal{U}$ is the control variable and $g: \mathcal{U} \rightarrow \mathbb{R}^{l}, g(u)=\left(g_{1}(u), \ldots, g_{l}(u)\right)^{\top}$ and $h: \mathcal{U} \rightarrow \mathbb{R}^{m}$, $h(u)=\left(h_{1}(u), \ldots, h_{m}(u)\right)^{\top}$, are inequality and equality constraints, respectively. The space of the control variables $\mathcal{U}$ is also called the decision space (according to the term decision variable for $u$ in classical multiobjective optimization), and the objective function maps $u$ to the objective space. Depending on the problem setup, $\mathcal{U}$ can either be finite-dimensional, i.e., $\mathcal{U}=\mathbb{R}^{n}$, or some appropriate function space. 
Remark 1. It is common in finite-dimensional optimization to use the notation $x$ for the control or optimization variable and $F$ for the objective function. In contrast to that, $u$ and $J$ are more common for control problems. In order to unify the notation, the latter will be used throughout this article for all optimization and optimal control problems.

In contrast to classical optimization, in optimal control, we have to compute an input in such a way that a dynamical system behaves optimally with respect to some specified cost functional. Hence, we have the system dynamics as an additional constraint, very often in the form of ordinary (ODEs) or partial differential equations (PDEs):

$$
\begin{aligned}
& \dot{y}(x, t)=G(y(x, t), u(t)), \quad(x, t) \in \Omega \times\left(t_{0}, t_{e}\right], \\
& a(x, t) \frac{\partial y}{\partial n}(x, t)+b(x, t) y(x, t)=c(x, t), \quad(x, t) \in \Gamma \times\left(t_{0}, t_{e}\right], \\
& y\left(x, t_{0}\right)=y_{0}(x), \quad x \in \Omega,
\end{aligned}
$$

where the domain of interest $\Omega \subset \mathbb{R}^{n_{x}}$ is a connected open set with spatial dimension $n_{x}$ and the boundary is denoted by $\Gamma=\partial \Omega$ with outward normal vector $n$. The coefficients $a(x, t), b(x, t)$ and $c(x, t)$ in the boundary condition are given by the problem definition. The operator $G$ is a partial differential operator describing the evolution of the system. The cost functional $\hat{J}: \mathcal{U} \times \mathcal{Y} \rightarrow \mathbb{R}^{k}$ of an optimal control problem consequently depends on the control $u$, as well as the system state $y$, which results in a multiobjective optimal control problem:

$$
\begin{gathered}
\min _{u \in \mathcal{U}, y \in \mathcal{Y}} \hat{J}(u, y)=\min _{u \in \mathcal{U}, y \in \mathcal{Y}}\left(\begin{array}{c}
\int_{t_{0}}^{t_{e}} C_{1}(y(x, t), u(t)) d t+\Phi_{1}\left(y\left(x, t_{e}\right)\right) \\
\vdots \\
\int_{t_{0}}^{t_{e}} C_{k}(y(x, t), u(t)) d t+\Phi_{k}\left(y\left(x, t_{e}\right)\right)
\end{array}\right) \\
\text { s.t. } \quad(\operatorname{PDE}) \\
g_{i}(y, u) \leq 0, \quad i=1, \ldots, l, \\
h_{j}(y, u)=0, \quad j=1, \ldots, m .
\end{gathered}
$$

There are articles on multiobjective optimal control that specifically address the implications of multiple objectives for optimal control [6,7]; see also [8] for a short survey of methods. However, for many problems, there exists a unique solution $y$ for every $u$ such that (MOCP) can be simplified by introducing a so-called control-to-state operator $\mathcal{S}: \mathcal{U} \rightarrow \mathcal{Y}$; see [9] for details. By setting $\hat{J}(u, y)=\hat{J}(u, \mathcal{S} u)=J(u)$, the problem is transformed into (MOP). For this reason, we will from now on only consider (MOP).

In the situation where $\mathcal{U}$ is a function space (i.e., in the case of optimal control), the problem can be numerically transformed into a high-, yet finite-dimensional problem in a direct solution method via discretization, cf. $[10,11]$. This results in a large number of control variables, which can be very challenging on its own in multiobjective optimization. If the system dynamics are governed by a PDE, then the spatial discretization of the state $y$ results in an even higher number of unknowns, which can easily reach several millions or more [12].

In contrast to single objective optimization problems, there exists no total order of the objective function values in $\mathbb{R}^{k}$ with $k \geq 2$ (unless they are not conflicting). Therefore, the comparison of values is defined in the following way [4]:

Definition 1. Let $v, w \in \mathbb{R}^{k}$. The vector $v$ is less than $w$ (denoted by $v<w$ ), if $v_{i}<w_{i}$ for all $i \in\{1, \ldots, k\}$. The relation $\leq$ is defined in an analogous way. 
A consequence of the lack of a total order is that we cannot expect to find isolated optimal points. Instead, the solution to (MOP) is the set of optimal compromises (also called the Pareto set or set of non-dominated points):

Definition 2. Consider the multiobjective optimization problem (MOP). Then:

1. a point $u^{*}$ dominates a point $u$, if $J\left(u^{*}\right) \leq J(u)$ and $J\left(u^{*}\right) \neq J(u)$.

2. a feasible point $u^{*}$ is called globally Pareto optimal if there exists no feasible point $u \in \mathcal{U}$ dominating $u^{*}$. The image $J\left(u^{*}\right)$ of a globally Pareto optimal point $u^{*}$ is called a globally Pareto optimal value. If this property holds in a neighborhood $U\left(u^{*}\right) \subset \mathcal{U}$, then $u^{*}$ is called locally Pareto optimal.

3. the set of non-dominated feasible points is called the Pareto set $\mathcal{P}_{S}$ and its image the Pareto front $\mathcal{P}_{F}$.

A consequence of Definition 2 is that for each point that is contained in the Pareto set (the red line in Figure 1a), one can only improve one objective by accepting a trade-off in at least one other objective. Figuratively speaking, in a two-dimensional problem, we are interested in finding the "lower left" boundary of the feasible set in objective space (cf. Figure 1b).

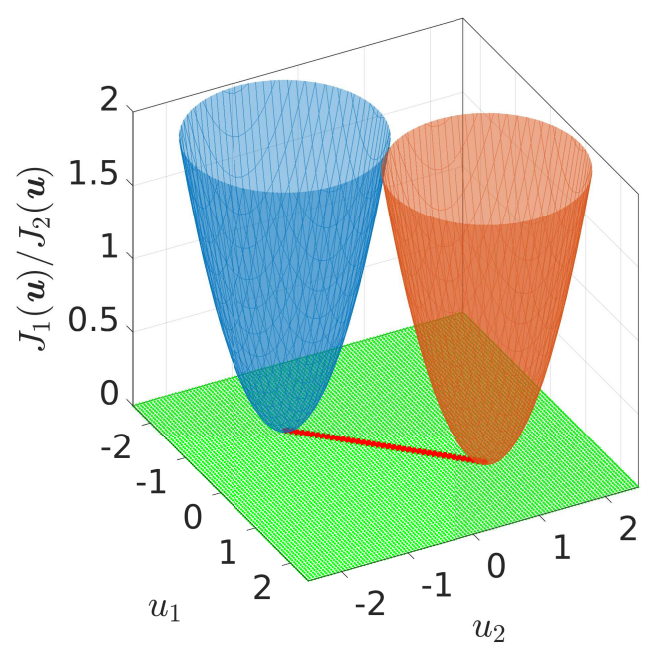

(a)

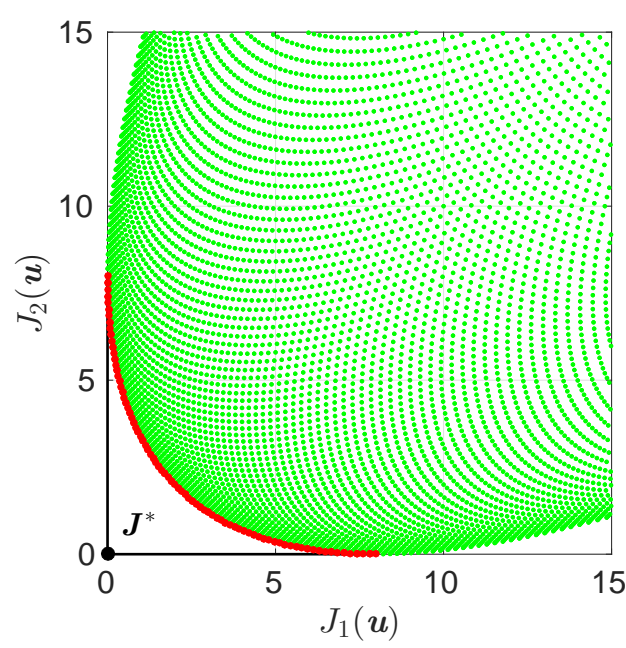

(b)

Figure 1. The red lines are the Pareto set (a) and Pareto front (b) of an exemplary multiobjective optimization problem (two paraboloids) of the form $\min _{u \in \mathbb{R}} J(u), J: \mathbb{R}^{2} \rightarrow \mathbb{R}^{2}$. The point $J^{*}=(0,0)^{\top}$ is called the utopian point.

Similar to single objective optimization, a necessary condition for optimality is based on the gradients of the objective functions. The first order conditions were independently discovered by Karush in 1939 [13] and by Kuhn and Tucker in 1951 [14]. Due to this, they are widely known as the Karush-Kuhn-Tucker (KKT) conditions:

Theorem 1 ([14]). Let $u^{*}$ be a Pareto-optimal point of Problem (MOP), and assume that $\nabla h_{j}\left(u^{*}\right)$ for $j=1, \ldots, m$ and $\nabla g_{s}\left(u^{*}\right)$ for $s=1, \ldots, l$ are linearly independent. Then, there exist non-negative scalars $\alpha_{1}, \ldots, \alpha_{k} \geq 0$ with $\sum_{i=1}^{k} \alpha_{i}=1, \gamma \in \mathbb{R}^{m}$ and $\mu \in \mathbb{R}^{l}$ such that: 


$$
\begin{aligned}
\sum_{i=1}^{k} \alpha_{i} \nabla J_{i}\left(u^{*}\right)+\sum_{j=1}^{m} \gamma_{j} \nabla h_{j}\left(u^{*}\right)+\sum_{s=1}^{l} \mu_{s} \nabla g_{s}\left(u^{*}\right) & =0, \\
h_{j}\left(u^{*}\right) & =0, \quad j=1, \ldots, m, \\
g_{s}\left(u^{*}\right) & \leq 0, \quad s=1, \ldots, l, \\
\mu_{s} g_{s}\left(u^{*}\right) & =0, \quad s=1, \ldots, l, \\
\mu_{s} & \geq 0, \quad s=1, \ldots, l .
\end{aligned}
$$

The set of points satisfying these conditions is called the set of substationary points $\mathcal{P}_{S \text {,sub }}$ [4]. Obviously, $\mathcal{P}_{S \text {,sub }}$ is a superset of the Pareto set $\mathcal{P}_{S}$. Many algorithms for MOPs compute the set of substationary points, in particular gradient-based methods, as we will see in the next section. This set can be reduced to the Pareto set in a consecutive step by performing a (comparatively inexpensive) non-dominance test.

\subsection{Solution Methods}

Many researchers in multiobjective optimization focus their attention on developing efficient algorithms for the computation of Pareto sets. Algorithms for solving MOPs can be compiled into several fundamentally different categories of approaches. The first category is based on scalarization techniques, where ideas from single objective optimization theory are extended to the multiobjective situation. All scalarization techniques have in common that the Pareto set is approximated by a finite set of Pareto-optimal points, which are computed by solving scalar subproblems. Consequently, the resulting solution methods involve solving multiple optimization problems consecutively. Scalarization can be achieved by various approaches such as the weighted-sum method, the $\epsilon$-constraint method, normal boundary intersection or reference point methods $[4,5,15]$.

Continuation methods make use of the fact that under certain conditions, the Pareto set is a smooth manifold of dimension $k-1$ [16]. This means that one can compute the tangent space in each point of the set, and a predictor step is performed in this space. The resulting point then has to be corrected to a Pareto-optimal solution using a descent method [17].

Another prominent approach is based on evolutionary algorithms [18,19], where the underlying idea is to evolve an entire population of solutions during the optimization process. Significant advances have been made concerning Multiobjective Evolutionary Algorithms (MOEAs) in recent years [20,21] (see also [22] for a survey) such that they are nowadays the most popular choice for solving MOPs due to the applicability to very complex problems and being easy to use in a black box fashion. Since convergence rates can be relatively slow for MOEAs, they can be coupled with locally fast methods close to the Pareto set. These approaches are known as memetic algorithms; see, e.g., [23-26].

Set-oriented methods provide an alternative deterministic approach to the solution of MOPs. Utilizing subdivision techniques, the desired Pareto set is approximated by a nested sequence of increasingly refined box coverings [27-29]. This way, a superset is computed, which converges to the desired solution, even in situations where the Pareto set is disconnected. However, their complexity depends on both the dimension of the Pareto set, as well as the decision space dimension. Due to this, one has to take additional steps to apply these algorithms for multiobjective optimal control problems.

Depending on the method of choice, gradient information can be used to accelerate convergence. While this is widely accepted in scalar-valued optimization, this is less the case when multiple objectives are present [30]. Nonetheless, many approaches exist where gradients are exploited, for example in order to create sequences converging to single points [31-33], to compute the entire set of valid decent directions $[30,34]$, to obtain superlinear or quadratic convergence $[35,36]$ or in combination 
with evolutionary approaches (memetic algorithms) [37-41]. In many of the gradient-based methods, the descent direction for all objectives is a convex combination of the individual gradients:

$$
q(u)=\sum_{i=1}^{k} \hat{\alpha}_{i} \nabla J_{i}(u)
$$

Here, $\hat{\alpha}$ is a fixed weight vector, which is determined in such a way that:

$$
\left\langle q(u), \nabla J_{i}(u)\right\rangle<0 \quad \forall i=1, \ldots, k,
$$

see, e.g., [31,32]. In the unconstrained case, there exists no $u$ satisfying (2) only if $\|q(u)\|=0$, which implies that $u$ is substationary, cf. (KKT).

\section{Surrogate Models}

The ever-increasing computational capabilities allow us to analyze more and more complicated systems with a very large number of degrees of freedom, also in the context of optimal control, where practical problems range from process control [42,43] and energy management [15] over space mission design [44] to mobility and autonomous driving [45-48].

The above-mentioned examples can all be described by ordinary differential equations with a finite-dimensional state space $\mathcal{Y}$. In contrast to these problems, many phenomena in physics such as mechanical strain, heat flow, electromagnetism, fluid flow or even multi-physics simulations are governed by partial differential equations. Using numerical discretization schemes for the approximation of the spatial domain (such as finite elements or finite volumes) results in a very large number of degrees of freedom and a heavy computational burden. For more complex systems (such as turbulent flows [49]), simulating the dynamics is already very costly. Consequently, optimal control of these systems is all the more challenging, and considering multiple objectives further increases the cost. Due to this reason, only a few problems have been addressed directly; see, e.g., [50-53]. A method exploiting the special structure in the system dynamics has been proposed in [54], and a special case of Pareto-optimal solutions, namely Nash equilibria, has been computed in [55,56] (When not using a priori selection methods such as scalarization or Nash equilibria, a decision maker selects the appropriate solution. This is called Multi-Criteria Decision Making (MCDM) [57] and is an entire area of research on its own. Thus, we will not go into further details about the decision making process here.).

A very popular approach to circumvent the problem of prohibitively large computational cost is the use of surrogate models. Here, the exact objective function $J(u)$ is replaced by a surrogate $J^{r}(u)$, where the superscript $r$ stands for reduced. In many situations, this surrogate function can be evaluated faster by several orders of magnitude. The challenge here is to find a good trade-off between acceleration and model accuracy, and many approaches have been proposed over the past two decades.

In optimal control, there are two fundamentally different ways for model reduction. The first case, which is equivalently applicable to multiobjective optimization problems, is to directly derive a surrogate for the objective function, i.e., $J^{r}: \mathcal{U} \rightarrow \mathbb{R}^{k}$ is constructed by polynomials, radial basis functions or other means. In optimal control, an alternative way of reducing the computational effort is by introducing a reduced model for the system dynamics:

$$
J^{r}(u)=\hat{J}^{r}(u, y)=\hat{J}\left(u, \mathcal{S}^{r} u\right)
$$

where the reduced control-to-state operator $\mathcal{S}^{r}$ indicates that the model reduction is due to a surrogate model for the system dynamics.

In both situations, we cannot expect that $J^{r}(u)=J(u)$ holds for all $u \in \mathcal{U}$. Instead, we introduce an error, which has to be taken into account. This is closely related to questions concerning uncertainty and 
noise. In this context, many researchers have addressed inaccuracies. In [58], the notion of $\epsilon$-efficiency (cf. Definition 3) was first introduced in order to handle uncertain objective values. Uncertainty has also been considered in the context of multiobjective evolutionary computation (see, e.g., [59-61]. Alternative). Methods such as probabilistic $[62,63]$, deterministic $[64]$ or set-oriented approaches $[65,66]$ have also been proposed. The special case of many-objective optimization is covered in [67], and applications are addressed in $[68,69]$. A different approach to uncertainties is via robust algorithms. Several examples from multiobjective optimization, as well as optimal control can be found in [43,70-72].

In the following, we will first introduce some results concerning inaccuracies in multiobjective optimization in Section 3.1 and then give an overview of existing methods for both of the above-mentioned approaches, i.e., surrogate models for the objective function (Section 3.2) or for the system dynamics (Section 3.3). Since the first approach has already been covered extensively in several surveys [1-3], we only give a brief overview of the existing methods and the corresponding references.

\subsection{Inaccuracies and $\epsilon$-Dominance}

When using surrogate models in order to accelerate the solution process, we have to accept an error both in the objective function, as well as the respective gradients. Furthermore, inaccuracies may occur due to stochastic processes or due to unknown model parameters. In these situations, the objective function and the corresponding gradients are only known approximately, which has to be taken into account.

Suppose now that we only have approximations $J^{r}(u), \nabla J^{r}(u)$ of the objectives $J_{i}(u)$ and the gradients $\nabla J_{i}(u), i=1, \ldots, k$, respectively. Furthermore, let us assume that upper bounds $\epsilon, \kappa \in \mathbb{R}^{k}$ for these errors are known:

$$
\begin{aligned}
\left\|J_{i}^{r}(u)-J_{i}(u)\right\|_{2} \leq \epsilon_{i} & \forall u \in \mathcal{U}, \\
\left\|\nabla J_{i}^{r}(u)-\nabla J_{i}(u)\right\|_{2} \leq \kappa_{i} & \forall u \in \mathcal{U} .
\end{aligned}
$$

In this situation, we need to replace the dominance property 2 by an inexact version, also known as $\epsilon$-dominance (see also $[58,69])$ :

Definition 3 ([66]). Consider the multiobjective optimization problem (MOP) where the objective function $J(u)$ is only known approximately according to (3). Then:

1. a point $u^{*}$ confidently dominates a point $u$, if $J^{r}\left(u^{*}\right)+\epsilon \leq J^{r}(u)-\epsilon$ and $J_{i}^{r}\left(u^{*}\right)+\epsilon_{i}<J_{i}^{r}(u)-\epsilon_{i}$ for at least one $i \in 1, \ldots, k$.

2. The set of almost non-dominated points, which is a superset of the Pareto set $\mathcal{P}_{S}$, is defined as:

$$
\mathcal{P}_{S, \epsilon}=\left\{u^{*} \in \mathcal{U} \mid \nexists u \in \mathcal{U} \text { which confidently dominates } u^{*}\right\}
$$

The concept of $\epsilon$-dominance is visualized in Figure 2. Theoretically, the true point could be anywhere inside the box defined by $\epsilon$ such that in the cases (a)-(c), the lower left point does not confidently dominate the other point. The necessary condition is violated for one component in (a) and (b), respectively, and for both components in (c). The gray points in Figure 2a show a possible realization of the true points in which no point is dominated by the other. In (d), the orange point confidently dominates the black one, and in (e), we see the implications for the computation of Pareto fronts. Due to the inexactness, the number of points that are not confidently dominated is larger than in the exact case. This is also evident in Figure 3, where the exact and the inexact solution of an example problem from production [32] have been computed with an extension of the subdivision technique presented in [27], cf. [66] for details. Here, inexactness is introduced due to uncertainties in pricing. $\epsilon$-dominance can be used for the development of algorithms for MOPs with uncertainties [59,61-63,65,67,68], for accelerating expensive MOPs [60,66,73], as well as for increasing the number of compromise solutions for the decision maker [63-65,69]. 
When considering gradient-based methods, inaccuracies in the gradients (Equation (4)) have to be taken into account. Since only approximations of the true gradient are known, these result in an inexact descent direction $q^{r}(u)$. The inaccuracy in the gradients introduces an upper bound for the angle between the individual gradients $\nabla J_{i}^{r}(u)$ and the descent direction $q^{r}(u)$. This is equivalent to a lower bound $\hat{\alpha}_{\min }$ for the weight vector, i.e., $\hat{\alpha}_{i} \in[0,1]$ in Equation (1) has to be replaced by $\hat{\alpha}_{i}^{r} \in\left[\hat{\alpha}_{\min , i}, 1\right]$ for $i=1, \ldots, k$ :

$$
q^{r}(u)=\sum_{i=1}^{k} \hat{\alpha}_{i}^{r} \nabla J_{i}^{r}(u)
$$

see [66] for a detailed discussion. The additional constraint ensures that if $q^{r}(u)$ is a descent direction for the inexact problem, it is also a descent direction for the true problem. Furthermore, we obtain a criterion for the accuracy up to which we can compute $\mathcal{P}_{S, \text { sub }}$ based on inexact gradient information.

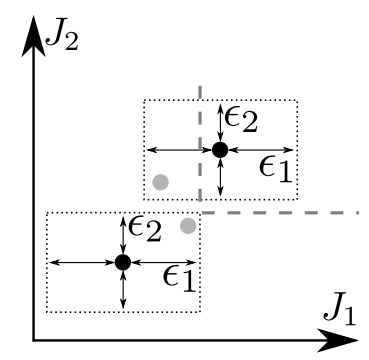

(a)

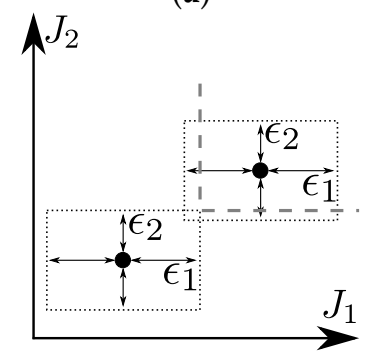

(c)

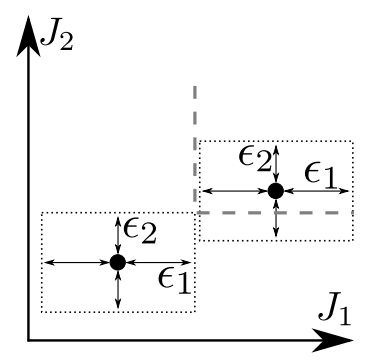

(b)

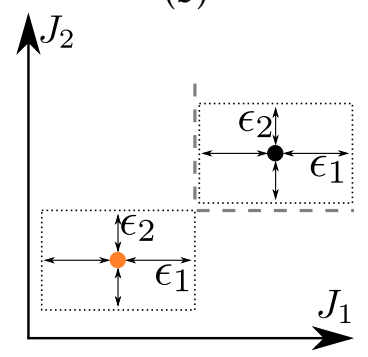

(d)

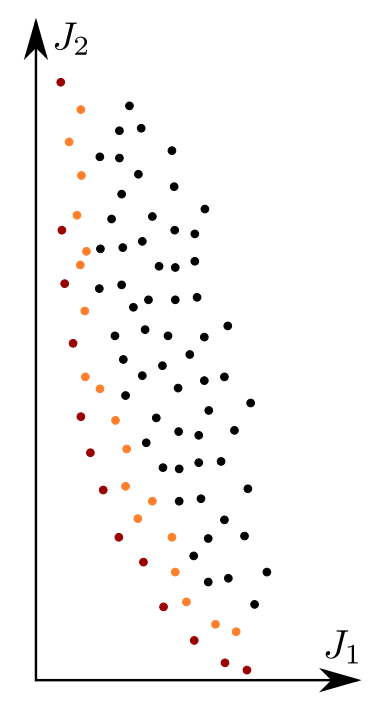

(e)

Figure 2. Example for the $\epsilon$-dominance property. A point-wise comparison is illustrated in (a)-(d). The uncertainties are marked by the dashed boxes. Only in case (d), the lower left point confidently dominates the other point. In $(\mathbf{e})$, the entirety of Pareto fronts for the exact problem $\left(\mathcal{P}_{F}\right)$ and the inexact problem $\left(\mathcal{P}_{F, \epsilon}\right)$ are shown in red and orange, respectively.

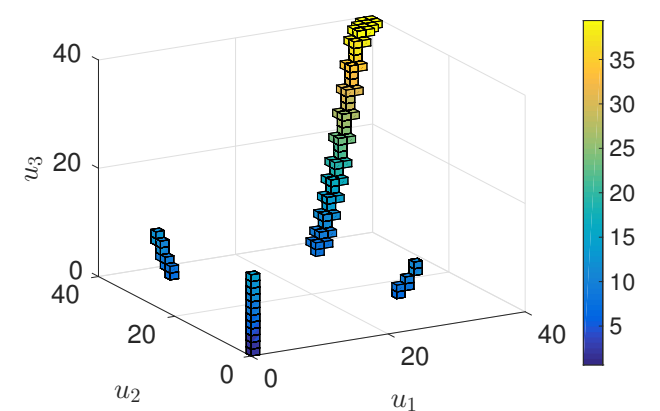

(a)

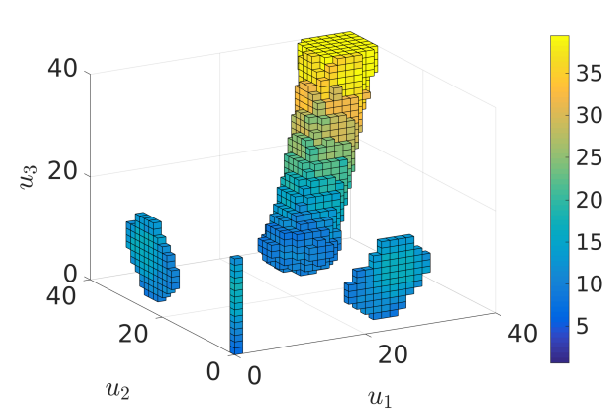

(b)

Figure 3. Example for an Multiobjective Optimization Problem (MOP) from production [32] where inexactness is introduced due to uncertainties in pricing. The Pareto set $\mathcal{P}_{S}$ for the exact problem is shown in (a), and the inexact set $\mathcal{P}_{S, \epsilon}$ is shown in (b). 
Theorem 2 ([66]). Consider the multiobjective optimization problem (MOP) without constraints. Only approximate gradients according to (4) are available, and consequently, the descent direction is also only known approximately according to Equation (6). Assume that $\left\|q^{r}(u)\right\|_{2} \neq 0$ and $\left\|\nabla J_{i}^{r}(u)\right\|_{2} \neq 0$ for $i=1, \ldots, k$. Let:

$$
\hat{\alpha}_{\min , i}=\frac{1}{\left\|\nabla J_{i}^{r}(u)\right\|_{2}^{2}}\left(\left\|q^{r}(u)\right\|_{2} \kappa_{i}-\sum_{\substack{j=1 \\ j \neq i}}^{k} \hat{\alpha}_{j}\left(\nabla J_{i}^{r}(u) \cdot \nabla J_{i}^{r}(u)\right)\right), \quad i=1, \ldots, k .
$$

Then, the following statements are valid:

(a) If $\sum_{i=1}^{k} \hat{\alpha}_{\min , i}>1$ then there exists no direction $q(u)$ with:

$$
\left\langle q(u), \nabla J_{i}(u)\right\rangle<0 \quad \forall i=1, \ldots, k,
$$

i.e., no descent direction for the exact problem.

(b) All points $u$ with $\sum_{i=1}^{k} \hat{\alpha}_{\min , i}=1$ are contained in the set:

$$
\mathcal{P}_{S, \kappa}=\left\{u \in \mathbb{R}^{n} \mid\left\|\sum_{i=1}^{k} \hat{\alpha}_{i} \nabla J_{i}(u)\right\|_{2} \leq 2\|\kappa\|_{\infty}\right\} \text {. }
$$

A combination of Theorem 2 with the subdivision algorithm from [27] is shown in Figure 4. The algorithm constructs a nested sequence of increasingly refined box coverings, which converges to the set of substationary points where in the unconstrained case, $\left\|q\left(u^{*}\right)\right\|=0$ holds for all $u^{*} \in \mathcal{P}_{S \text {,sub }}$. The set $\mathcal{P}_{S \text {,sub }}$ is shown in red in Figure $4 \mathrm{a}$. Due to the inexactness, we can no longer guarantee $\left\|q\left(u^{*}\right)\right\|=0$. Instead, we obtain the set $\mathcal{P}_{S, k}$, which is shown in green. The background is colored according to the optimality condition $\|q(u)\|$ of the exact problem, and the dashed white line indicates the error bound (7) from the above theorem.

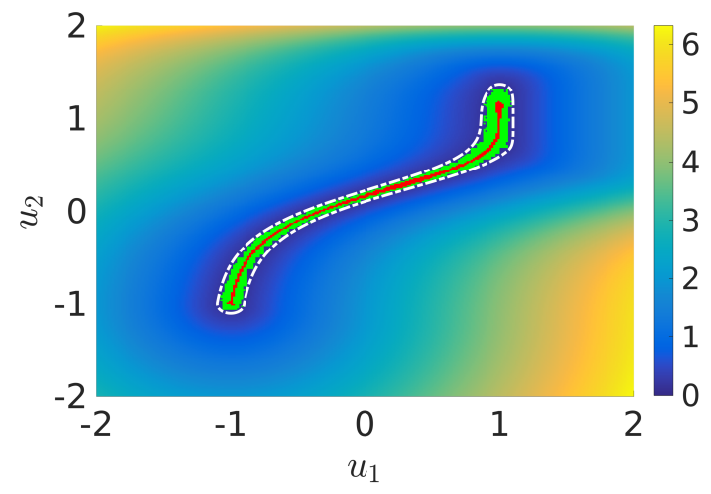

(a)

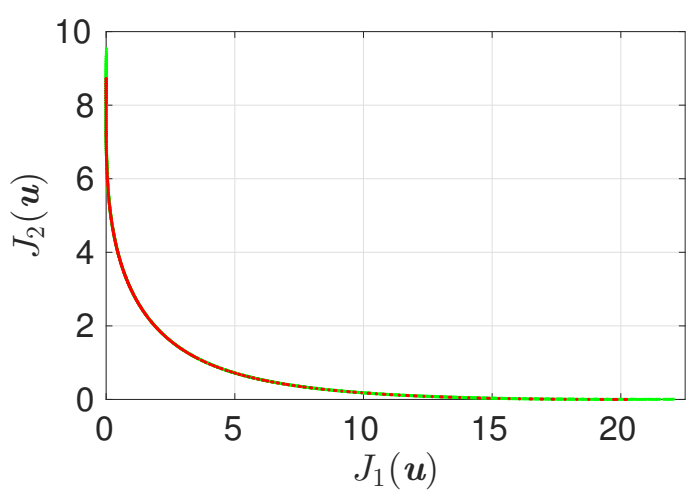

(b)

Figure 4. Exact and inexact solutions $\left(\mathcal{P}_{S}\right.$ and $\left.\mathcal{P}_{S, k}\right)$ for a simple example with $J: \mathbb{R}^{2} \rightarrow \mathbb{R}^{2}$, cf. [66] for details. (a) The sets $\mathcal{P}_{S}$ and $\mathcal{P}_{S, \kappa}$ (for a random error with $\kappa=(0.01,0.01)^{\top}$ ) are shown in red and green, respectively. The background is colored according to the optimality condition $\|q(u)\|$, which has to be zero for all substationary points. The dashed white line shows the error bound as derived in Theorem 2. (b) The corresponding Pareto fronts.

\subsection{Surrogate Models for the Objective Function}

The most straight-forward approach for introducing a surrogate model is to directly construct a map from the control to the decision space. This means that only the essential input-output behavior is covered, whereas internal states, as well as the system dynamics are neglected. Using such a meta 
model, one can very quickly obtain the objective function value for every $u$. This approach is equally applicable in finite-dimensional multiobjective optimization and has been used extensively in this context. To this end, we will only briefly cover the main questions that have to be addressed when using such models.

In special cases, one can exploit some structure in the problem formulation that yields a simplified analytic expression. However, in most cases, even if the equations can be written down in closed form, this approach requires a deep understanding of the underlying system, which is often hard to obtain. Moreover, small changes in the problem setup may result in having to repeat this tedious process all over. Due to these reasons, data-based approaches are used much more frequently. They are often easy to apply and much more general. In these approaches, the original objective function (or even a real-world experiment) is evaluated for a small number of inputs that are contained in the set $\mathcal{U}_{\text {ref }}=\left\{u^{1}, \ldots, u^{p}\right\}$, where $p$ is the number of function evaluations (or experiments). The data points $J\left(u^{1}\right)-J\left(u^{p}\right)$ are then used to fit the meta model such as, e.g., the coefficients of a polynomial basis. Obviously, the choice of suitable ansatz functions is essential for the success of a meta modeling strategy. Popular choices are:

- Response Surface Models (RSM);

- Radial Basis Functions (RBF);

- $\quad$ statistical models such as Kriging or Gauss regression;

- machine learning methods such as artificial neural networks or support vector machines;

See [1] for an extensive survey in the context of multiobjective optimization. Additional surveys can be found in [74], where different statistical methods are compared, in [3,75] in relation to MOEAs or in [76], where RSM and RBF are compared for crashworthiness problems.

Besides selecting the correct meta model, questions concerning the training dataset have to be answered:

1. How large does the set $\mathcal{U}_{\text {ref }}$ have to be?

2. How can we pick the correct elements for $\mathcal{U}_{\text {ref }}$ ?

3. Do we define $\mathcal{U}_{\text {ref }}$ in advance or online during the model building process?

In addition to the meta model, Point 1 significantly depends on the problem under consideration. The more non-linear a problem is, the more data points are generally required to accurately construct a meta model. Obviously, the number also depends on Point 2 , the locations $u^{1}-u^{k}$ of these evaluations. The question of choosing the correct location is closely related to the field of optimum experimental design or Design of Experiments (DoE); see, e.g., [77,78]. The relevant question there is how to optimally pick a set of experiments such that the overall approximation error becomes minimal. Such approaches have successfully been coupled with multiobjective optimization in [79,80]. Point 3 depends both on the meta modeling approach, as well as the problem under consideration. In some situations (e.g., for real-world experiments), it may not be possible to iteratively determine the experiments. Instead, a batch approach has to be used. In computer experiments, flexibility is often higher such that an interplay between model building and high-fidelity evaluations can help to further reduce the number of experiments. In the context of machine learning, this process is also known as active learning $[81,82]$.

Many algorithms combining multiobjective optimization and meta modeling have been proposed, and there exists a vast literature concerning this topic. Table 1 on page 17 mentions both survey articles and a (non-exhaustive) selection of popular methods.

\subsection{Surrogate Models for the Dynamical System}

The above-mentioned meta modeling methods are widely studied and have been successfully applied in a large variety of multiobjective optimization problems. In the context of control, there exists an alternative option, which is to derive a surrogate model for the system dynamics, i.e., to replace the high-fidelity control-to-state operator $\mathcal{S}$ by a less expensive surrogate model $\mathcal{S}^{r}$. Since the largest part 
of the computational effort is due to solving the dynamical system, by this, the reduced cost function $J^{r}(u)=\hat{J}^{r}(u, y)=\hat{J}\left(u, \mathcal{S}^{r} u\right)$ is also much less expensive to evaluate.

The use of Reduced Order Models (ROMs) is not limited to control, but has been used successfully in a large variety of multi-query problems [12], and several extensive surveys have been written on reduced order modeling and using ROMs in prediction, uncertainty quantification or optimization; see [83-85]. For nonlinear problems, the two most widely-used approaches are the reduced basis method and Proper Orthogonal Decomposition (POD) (also known as Principal Component Analysis (PCA) or Karhunen-Loève decomposition).

\subsubsection{ROMS via Proper Orthogonal Decomposition or the Reduced Basis Method}

Numerically solving a PDE is generally realized by discretizing the spatial domain with a numerical mesh using finite differences, finite elements or finite volumes. By this, the infinite-dimensional state space $\mathcal{Y}$ is transformed into a finite-dimensional space $\mathcal{Y}^{N}$ via Galerkin projection:

$$
y(x, t) \approx y^{N}(x, t)=\sum_{i=1}^{N} z_{i}^{N}(t) \phi_{i}(x) .
$$

Here, $N$ denotes the number of degrees of freedom, and $\phi_{i}(x)$ are basis functions with local support such as indicator functions or hat functions. This transforms the PDE into an $N$-dimensional ordinary differential equation for the coefficients $z$. For complex domains, as well as complex dynamics, the dimension can easily reach the order of millions such that solving the problem in $\mathcal{Y}^{N}$ can quickly become very expensive, which is particularly challenging in the multi-query case.

The general concept in projection-based model reduction is therefore to find an appropriate space $\mathcal{Y}^{r}$ with dimension $\ell \ll N$ in which the system dynamics can nevertheless be approximated with sufficient accuracy. The two most common approaches to do this are the Reduced Basis (RB) method and Proper Orthogonal Decomposition (POD). In both cases, we compute $s$ so-called snapshots of the high-dimensional system and then use the dataset $\left\{y_{1}^{N}, \ldots, y_{s}^{N}\right\}$ to construct a reduced basis $\psi=\left\{\psi_{1}, \ldots, \psi_{\ell}\right\}$ such that:

$$
y(x, t) \approx y^{N}(x, t) \approx y^{r}(x, t)=\sum_{i=1}^{\ell} z_{i}^{\ell}(t) \psi_{i}(x)
$$

The following example describes this approach in more detail. For an extensive introduction, the reader is referred to $[86,87]$.

Example 1 (Heat equation). Suppose we want to solve the time-dependent heat equation on a domain $\Omega$ with homogeneous Neumann conditions on the boundary $\Sigma$ :

$$
\begin{aligned}
y_{t}(x, t)-\lambda \Delta y(x, t) & =0, & (x, t) & \in \Omega \times\left(t_{0}, t_{e}\right], \\
y(x, 0) & =y_{0}(x), & x & \in \Omega, \\
y_{n}(x, t) & =0, & (x, t) & \in \Sigma \times\left(t_{0}, t_{e}\right] .
\end{aligned}
$$

Here, $y$ is the temperature, and $\lambda$ is the heat conductivity. The subscripts $t$ and $n$ indicate the derivatives with respect to time and the outward normal vector of the boundary, respectively. We now derive the weak form of (9) by multiplying with a test function $\varphi$ and integrating over the domain $\Omega$. Using Gauss's theorem, we obtain the following equation:

$$
\int_{\Omega} y_{t}(\cdot, t) \varphi-\lambda \nabla y(\cdot, t) \cdot \nabla \varphi d x=0, \quad(x, t) \in \Omega \times\left(t_{0}, t_{e}\right] .
$$


If we want to solve (10) using the finite element method, we have to insert the Galerkin ansatz (8) into (10) and individually take each of the basis functions as a test function. By this, we obtain the following system of equations:

$$
\int_{\Omega} \sum_{i=1}^{N} z_{i, t}^{N}(t) \phi_{i} \phi_{j}-\lambda z_{i}^{N}(t) \nabla \phi_{i} \cdot \nabla \phi_{j} d x=0, \quad j=1, \ldots, N .
$$

Introducing the mass matrix $M \in \mathbb{R}^{N \times N}$ and the stiffness matrix $K \in \mathbb{R}^{N \times N}$ with:

$$
M_{i, j}=\int_{\Omega} \phi_{i} \phi_{j} d x, \quad K_{i, j}=\int_{\Omega} \nabla \phi_{i} \cdot \nabla \phi_{j} d x,
$$

this yields the following $\mathrm{N}$-dimensional linear system:

$$
M z_{t}^{N}(t)-\lambda K z^{N}(t)=0 .
$$

If we now want to compute a reduced order model instead of a high-dimensional finite element approximation, we can apply the same procedure, except that now, we have to use the reduced basis in the Galerkin ansatz, as well as for test functions.

The most important difference between RB and POD is the area of application, although this is not a strict separation. RB is mostly applied to parameter-dependent, yet time-independent (i.e., elliptic) problems, whereas POD (introduced in [88]) is applied to time-dependent problems described by parabolic or hyperbolic PDEs. Consequently, in RB, the snapshots $\left\{y_{1}^{N}, \ldots, y_{s}^{N}\right\}$ are solutions corresponding to parameters $\left\{u_{1}, \ldots, u_{s}\right\}$, and in POD, they are snapshots in time, collected at the time instants $\left\{t_{0}, \ldots, t_{s-1}\right\}$. Using an equidistant time grid $h$, the snapshots are taken at $\left\{t_{0}, t_{0}+h \ldots, t_{0}+(s-1) h\right\}$. In RB, the snapshots $\left\{y_{1}^{N}, \ldots, y_{s}^{N}\right\}$ often directly serve as the basis $\psi$. For time-dependent problems, this can cause numerical difficulties since some snapshots might be very similar (e.g., for very slow systems or periodic dynamics) such that the snapshot matrix $S=\left(y_{1}^{N}, \ldots, y_{s}^{N}\right)$ is ill-conditioned. Due to this, a singular value decomposition is performed on $S$, and the $\ell$ leading left singular vectors are taken as the basis $\psi$. This results in an orthonormal basis, which can be shown to be optimal with respect to the $L^{2}$ projection error $[87,88]$. Furthermore, the truncation error is given by the sum over the neglected singular values:

$$
\epsilon_{\ell}=\frac{\sum_{i=\ell+1}^{s} \sigma_{i}}{\sum_{j=1}^{s} \sigma_{j}}
$$

Whereas the error between the infinite-dimensional solution and the solution via a standard discretization approach can be neglected in many situations, the error of the ROM depends on several factors such as the reference data, the basis size and the parameter or control for which the ROM is evaluated. Consequently, this error can be significant such that proper care has to be taken. The most common approach is to derive bounds either for the error of the reduced state $\left\|y^{r}-y^{N}\right\|$ or, in the case of optimal control, of the optimal solution $\left\|u^{r}-u^{N}\right\|$ obtained using the ROM; see, e.g., [89-93] for POD or [94-97] for RB methods. In addition, there are other measures that can be taken such as deriving balanced input-output behavior [98,99] or introducing additional terms [100] or modifications [101] in the POD-based ROM. For more detailed introductions to RB and POD, the reader is referred to [97] and $[87,88]$, respectively.

\subsubsection{Optimal Control Using Surrogate Models}

There is a rich literature on optimal control of PDEs using surrogate models. The approaches can be summarized into three main categories: 
1. build a model once,

2. construction of regular updates in a trust region approach,

3. construction of regular updates using error estimators.

Whereas the first category is the most efficient one (see, e.g., [102] for optimal control of the Navier-Stokes equations), it is in general not possible to prove the convergence of the resulting algorithm.

In the second approach (which was developed by Fahl [103] for POD-based ROMs and one objective), one defines a trust region within which the current surrogate model is considered as trustworthy; see Figure 5 for an illustration. The ROM-based optimal control problem is then solved with the additional constraint that the solution has to remain within the trust region, i.e., $\left\|u^{i}-u^{\text {ref }}\right\| \leq \delta^{i}$, where $i$ is the current step of the iterative optimization scheme and $\delta^{i}$ is the current trust region radius. After having obtained $u^{i}$, the high-dimensional system is evaluated, and the improvement of the full system is determined:

$$
\rho=\frac{\left|J^{N}\left(u^{i}\right)-J^{N}\left(u^{i-1}\right)\right|}{\left|J^{r}\left(u^{i}\right)-J^{r}\left(u^{i-1}\right)\right|} .
$$

If $\rho$ is close to one, then the ROM is sufficiently accurate, and the iterate $u^{i}$ is accepted. We then use the high-dimensional solution to construct the next ROM at $u^{\text {ref }}=u^{i}$. If, on the other hand, $\rho$ is close to zero, then the ROM accuracy was bad, and the iterate $u^{i}$ is rejected. Instead, the trust region radius $\delta^{i}$ is reduced, and the optimal control problem is solved again. Using the Trust Region (TR-POD) approach, one can ensure convergence to the optimal solution of the high-dimensional problem. In the case of the Navier-Stokes equations, this has been shown for different problem setups $[103,104]$.

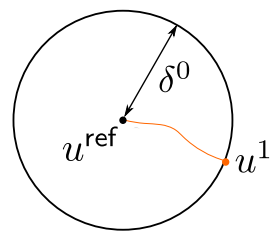

(a)

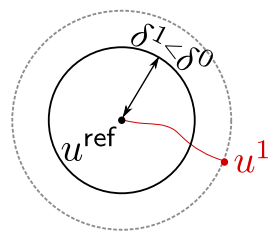

(b)

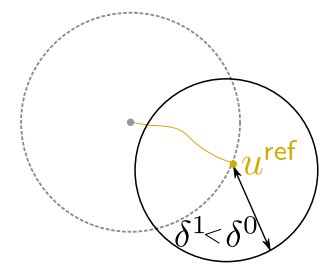

(c)

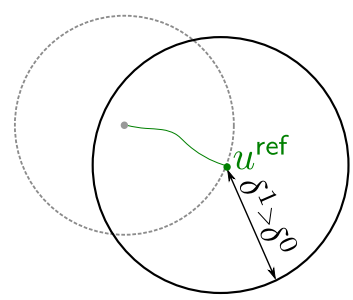

(d)

Figure 5. Trust region method. (a) The Reduced Order Model (ROM)-based optimal control problem is solved within the trust region $\delta^{0}$. (b) If the improvement is poor for the full system (i.e., $\rho$ is small), then the trust region radius is reduced, and we repeat the computation with the same problem. (c) If the improvement is acceptable (intermediate values of $\rho$ ), then we compute a new model and proceed with a smaller trust region $\delta^{1}<\delta^{0}$. (d) If the improvement is good (i.e., $\rho \approx 1$ ), then the trust region radius is increased.

In the third approach, error estimators $\Delta^{J}$ for the current iterate $u^{i}$ are required. By evaluating $\Delta^{J}\left(u^{i}\right)$, it is possible to efficiently estimate the error between the high- and the low-dimensional solution, since:

$$
\left|J^{r}\left(u^{i}\right)-J^{N}\left(u^{i}\right)\right|<\Delta^{J} .
$$

If this error estimate is larger than some prescribed upper bound $\epsilon$, then the ROM has to be updated using data from a high-dimensional solution. Detailed information on error estimates can be found in, e.g., [91-97].

\subsection{ROM-Based Multiobjective Optimal Control of PDEs}

All three approaches for using ROMs in optimal control have recently been extended to multiobjective optimal control problems. Besides different ROM techniques, different algorithms 
for MOPs have been used, as well, such that a variety of methods has evolved, each of which is well suited for certain situations.

\subsubsection{Scalarization}

A natural and widely-used approach to MOPs is via scalarization. By this, the vector of objectives is synthesized into a scalar objective function, and the MOP is transformed into a sequence of scalar optimization problems for different scalarization parameters. In terms of ROM-based optimal control, this is advantageous because many techniques from scalar-valued optimal control can be extended. The main difference is now that the objective function may have a more complicated structure. In $[105,106]$, the weighted sum method has been used in combination with RB in order to solve MOPs constrained by elliptic PDEs. In the weighted sum method, scalarization is achieved via convex combination of the individual objectives using the weight vector $\alpha$ :

$$
\min _{u \in \mathcal{U}} \bar{J}(u)=\min _{u \in \mathcal{U}} \sum_{i=1}^{k} \alpha_{i} J_{i}(u)
$$

The weighted sum method is probably the most straight-forward approach for including ROMs in MOPs. However, the method has strong limitations in the situation of non-convex problems, where it is impossible to compute the entire Pareto set [5].

A more advanced approach is the so-called reference point method [5] (cf. Figure 6 for an illustration), where the distance to an infeasible target point $T$ with $T<J(u)$ has to be minimized:

$$
\min _{u \in \mathcal{U}} \bar{J}(u)=\min _{u \in \mathcal{U}}\left\|T-J_{i}(u)\right\|
$$

By adjusting the target, we can move along the Pareto front and hence obtain an approximately equidistant covering of the front. The reference point method has been coupled with all three of the above-mentioned ROM approaches. In [107], it was used for multiobjective optimal control of the Navier-Stokes equations using one reduced model (cf. Figure 7a). Here, the objectives are to stabilize a periodic solution (the well-known von Kármán vortex street) and to minimize the control cost at the same time. In [73], the trust region framework by Fahl (TR-POD, [103]) was extended (cf. Figure 7b-c for a heat flow problem with a tracking and a cost minimization objective). The third ROM approach was used in [108,109]. The difficulty here is that the minimization of the distance to the target point results in a more complicated objective function, which has to be treated carefully.

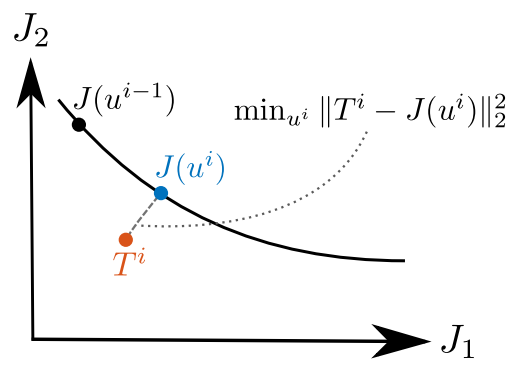

(a)

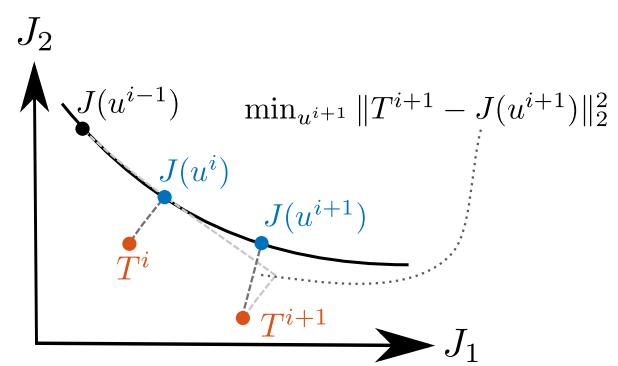

(b)

Figure 6. Reference point method. (a) Determination of a Pareto-optimal solution by solving (13). (b) Determination of the consecutive point on the Pareto front by adjusting the target and solving the next scalar problem.

Scalarization techniques generally have the same limitations on the decision space dimension as scalar-valued optimal control problems. This means that very efficient techniques (both direct and indirect) exist for high-dimensional controls. However, the number of objectives is limited because the 
parametrization in the scalarization step becomes extremely tedious, and it is almost impossible to obtain a good approximation of the entire Pareto set for more than three objectives.

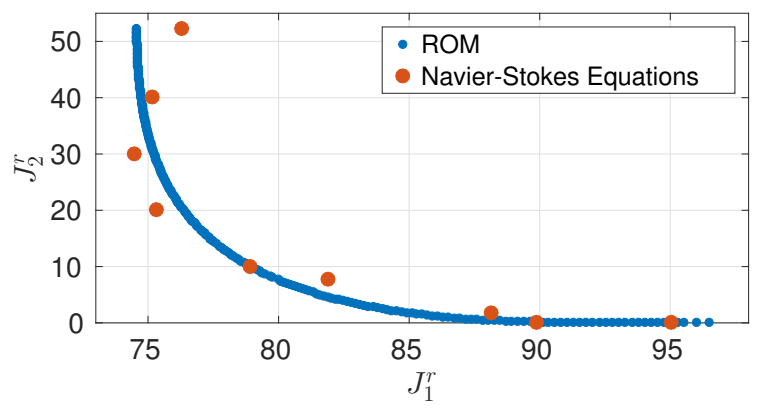

(a)

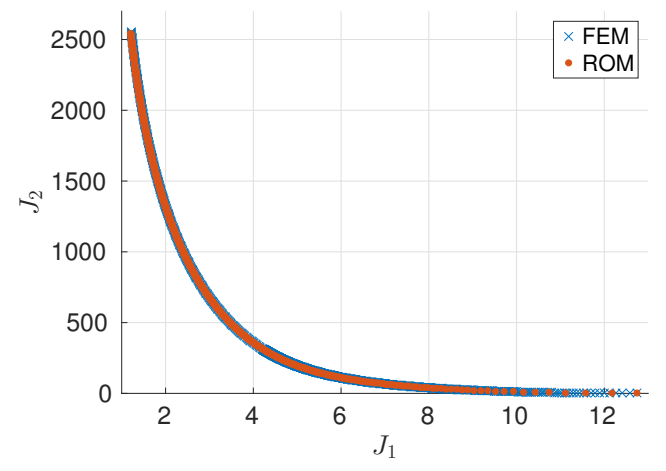

(b)

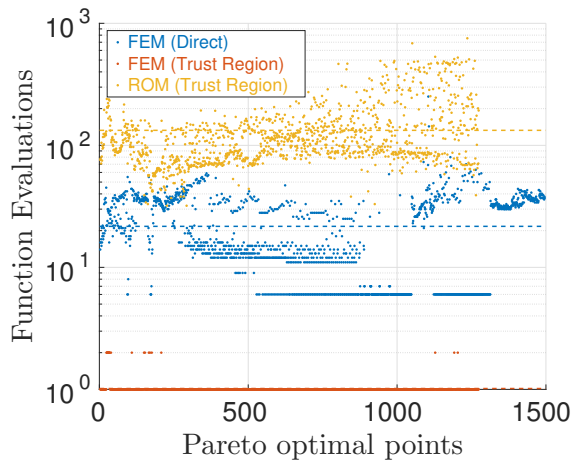

(c)

Figure 7. (a) Pareto front for an MOCP involving the Navier-Stokes equations (flow stabilization vs. cost), solved by coupling of an ROM (created once in advance) with the reference point method. Although we observe acceptable agreement, convergence cannot be guaranteed. (b) Pareto front for a heat flow MOCP (reference tracking vs. cost), solved by the TR-POD approach coupled with the reference point method. Convergence is achieved while reducing the number of expensive finite element (FEM) evaluations by a factor of $\approx 22$, cf. (c).

\subsubsection{Set-Oriented Approaches with $\epsilon$-Dominance}

In contrast to scalarization, in set-oriented techniques, the Pareto set is approximated by a box covering [27-29]. Here, the limitations are contrary, meaning that the dimension of the decision space is rather limited, while the number of objectives does not pose any problems for the algorithms. In practice, however, the computational cost increases exponentially with the number of objectives (i.e., the dimension of the Pareto set) such that we are still limited to a moderate number of objectives.

First results coupling the subdivision algorithm developed in [27] with error estimates for POD-based ROMs have recently appeared [110,111]. In the subdivision algorithm, the decision space is divided into boxes, which are alternatingly subdivided and selected. In the subdivision step, each existing box is subdivided into two smaller boxes. In the selection step, all boxes are eliminated that are dominated, i.e., they do not cover any part of the Pareto set. Numerically, this is realized by representing a box by a finite number of sample points and then marking a box as dominated if all sample points are dominated by samples from another box in the covering; see [27] for details.

The subdivision algorithm can be extended to inaccuracies by replacing the strict dominance test by an $\epsilon$-dominance test as presented in Section 3.1 (see also Figure 3). After fixing an upper bound $\epsilon$, we have to ensure that the surrogate models we use do not violate this bound anywhere in the control domain. Since this cannot be achieved with a single ROM, one has to use multiple, locally valid ROMs instead (cf. Figure 8c). The covering by local ROMs is managed in such a way that all points 
in the neighborhood around the reference $u^{\text {ref }}$ at which the data for the ROM were collected satisfy the prescribed error bound $\epsilon$. This way, the number of solutions of the high-dimensional system can be reduced significantly. A comparison between the exact and the ROM-based solution is shown in Figure 8 for a semilinear heat flow MOCP with two tracking type objectives and a cost minimization objective. Due to the ROM approach, the number of evaluations of the FEM model could be reduced by a factor of $\approx 1000$.

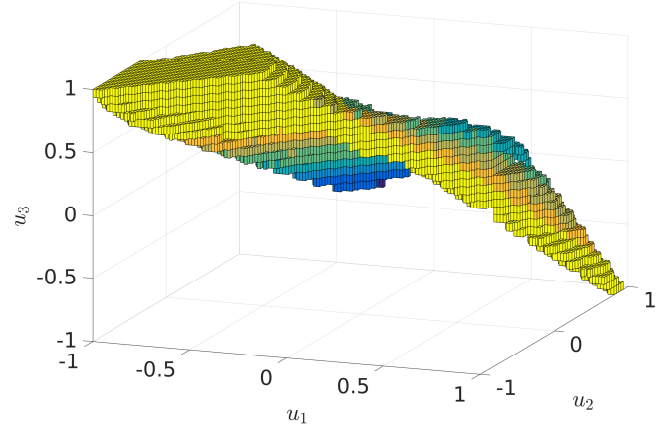

(a)

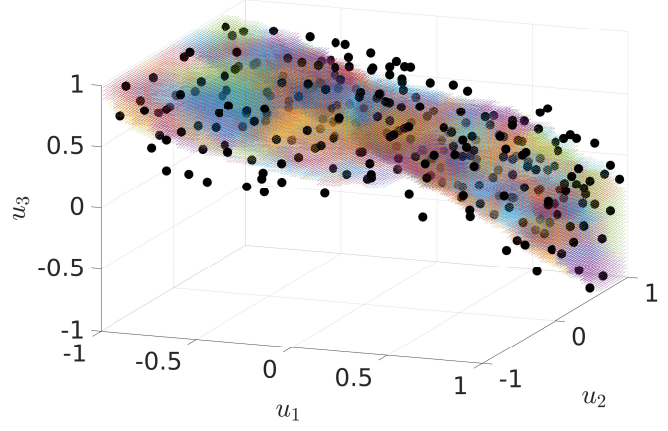

(c)

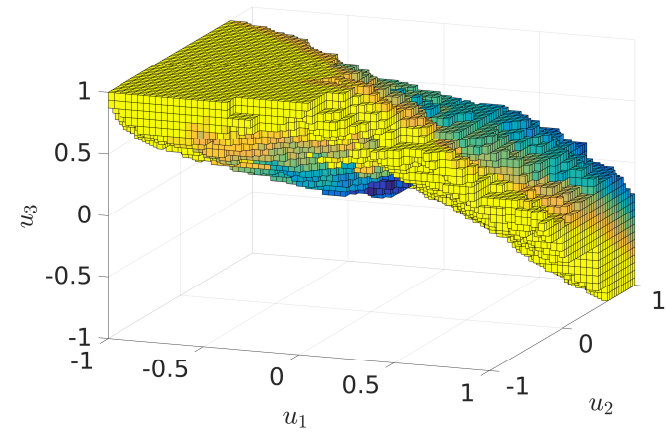

(b)

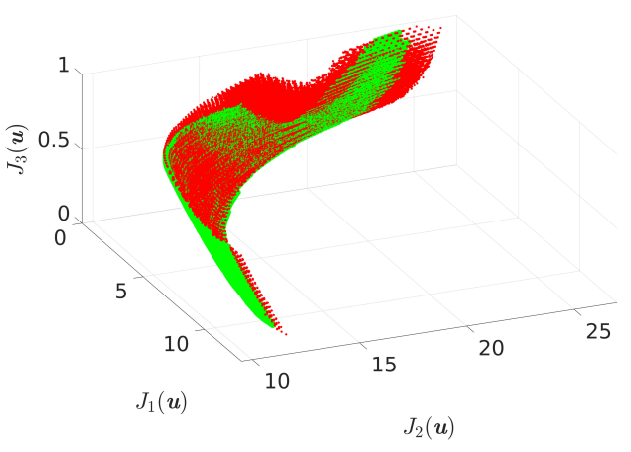

(d)

Figure 8. (a) Pareto set of a semilinear heat flow MOP with four controls (coloring according to $u_{4}$ ), solved directly including an FEM model in the subdivision algorithm. (b) Pareto set of the same problem, solved with localized ROMs. (c) The reference controls for which the local ROMs have been computed are shown in black, and the colored dots are sample points at which the objective function was evaluated. The colorings denote assignments to a specific ROM. (d) The corresponding Pareto fronts, where the FEM solution is shown in green and the ROM solution in red.

\subsection{Summary}

Before moving on to feedback control, a summary of the relevant publications where multiobjective optimization and meta modeling interact is given in Table 1 . The references are categorized into surveys, algorithms using surrogate models for the objective function, the system dynamics and specific reduction approaches for MOPs. Furthermore, some applications are referenced. 
Table 1. Overview of publications (in chronological order) where surrogate modeling and multiobjective optimization are combined. MOEA, Multiobjective Evolutionary Algorithm; RSM, Response Surface Model; POD, Proper Orthogonal Decomposition; TR, Trust Region.

\begin{tabular}{|c|c|}
\hline \multicolumn{2}{|r|}{ Surveys } \\
\hline Tabatabei et al. [2], Chugh et al. [3] & Extensive surveys on meta modeling for MOEAs \\
\hline $\begin{array}{l}\text { Voutchkov and Keane [74], } \\
\text { Knowles and Nakayama [1], Jin [75] }\end{array}$ & $\begin{array}{l}\text { Surveys on meta modeling approaches from statistics (RSM, } \\
\text { RBF) and machine learning in combination with MOEAs }\end{array}$ \\
\hline $\begin{array}{l}\text { Benner et al. [83], Taira et al. [85], } \\
\text { Peherstorfer et al. [84] }\end{array}$ & Surveys on reduced order modeling of dynamical systems \\
\hline \multicolumn{2}{|c|}{ Algorithms Using Meta Models for the Objective Function } \\
\hline Ong et al. [112], Ray et al. [113] & Combination of RBF and MOEA \\
\hline Chung and Alonso [114], Keane [115] & Combination of kriging models and MOEA \\
\hline Karakasis and Giannakoglou [116] & $\mathrm{RBF}$ as an inexpensive pre-processing step in a MOEA \\
\hline Knowles [117] & Combination of DoE and an interactive method \\
\hline Zhang et al. [118] & Combination of Gaussian process models and scalarization \\
\hline Telen et al. [79] & Combination of DoE and scalarization and MOEA \\
\hline Chugh et al. [119] & $\begin{array}{l}\text { Kriging model in combination with reference vector approach for } \\
\text { MOPs }\end{array}$ \\
\hline \multicolumn{2}{|c|}{ Meta Models Specifically Tailored to Multiobjective Optimization } \\
\hline Shimoyama et al. [120] & Kriging surrogate for hypervolume approximation (MOEA) \\
\hline Pan et al. [121] & Surrogate model for dominance relations with uncertainties \\
\hline \multicolumn{2}{|c|}{ Algorithms Using Surrogate Models for the System Dynamics } \\
\hline Iapichino et al. [105] & Combination of POD and weighted sum \\
\hline Banholzer et al. [108,109] & Combination of POD and reference point method \\
\hline Iapichino et al. [106] & Combination of RB and weighted sum \\
\hline Peitz [73] & Combination of TR-POD and reference point method \\
\hline Beermann et al. $[110,111]$ & Combination of POD and set-oriented method \\
\hline \multicolumn{2}{|r|}{ Applications } \\
\hline Albunni et al. [53] & POD and MOEA applied to the Maxwell equation \\
\hline Ma and $\mathrm{Qu}[80]$ & $\begin{array}{l}\text { MO of a switched reluctance motor by coupling RSM and } \\
\text { MOEA (particle swarm optimization) }\end{array}$ \\
\hline Peitz et al. [107] & $\begin{array}{l}\text { POD-based multiobjective optimal control of the Navier- } \\
\text { Stokes equations via scalarization and set-oriented methods }\end{array}$ \\
\hline Wang et al. [122] & $\begin{array}{l}\text { MOEA with multi-fidelity surrogate-management and } \\
\text { offline-online decomposition applied to a trauma system }\end{array}$ \\
\hline
\end{tabular}

\section{Feedback Control}

Even when the objective function is not very expensive to evaluate, MOCPs often have a large computational cost; see, e.g., [123] for various examples. This becomes a limiting factor in situations where the solution time is critical as is the case in real-time applications. Due to the increasing computational power, as well as the advances in algorithms, Model Predictive Control (MPC) (see [124,125] for extensive introductions) has become a very powerful and widely-used method for realizing model-based feedback control of complex systems.

In MPC, an optimal control problem is solved in a short time horizon (the prediction horizon) while the real system (the plant) is running. Then, the first entry of the optimal control is applied to the plant, and the process is repeated with the time frame moving forward by one sample time $h$; 
see Figure 9 for an illustration. This way, a closed-loop behavior is achieved. On the downside, we have to solve the optimal control problem within the sample time $h$. This can be in the order of seconds or minutes (in the case of chemical processes) down to a few microseconds, for example in power electronics applications.

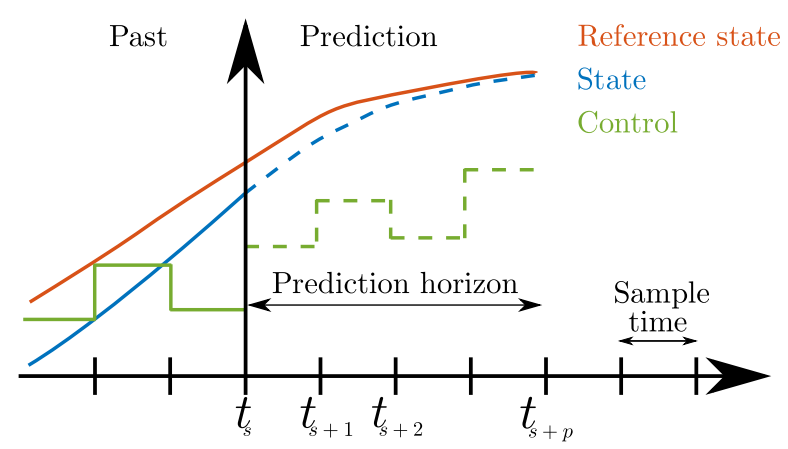

Figure 9. Sketch of the MPC method. Due to the real-time constraints, the optimization problem has to be solved faster than the sample time $h$.

In many MPC problems, stabilization of the system with respect to some reference state is the most important aspect. Nevertheless, there exist variations where stability is not an issue such that other (more economic) objectives can be pursued. These methods are known as economic MPC [126,127]. Another variation is the so-called explicit MPC [128], where the optimal control is computed in advance for a large number of different states and stored in a library. This way, the computational effort is shifted to an offline phase, and during operation, we only have to select the optimal control from the library.

As in multiobjective optimal control, there are numerous applications where multiple objectives are interesting in feedback control. This means that we would have to solve the problem (MOCP) with $t_{0}=t_{s}$ and $t_{e}=t_{s+p}$ repeatedly within the sample time $h$. It is immediately clear that even the simplest MOCPs cannot be solved fast enough to allow for real-time applicability. Consequently, efficient algorithms have to be developed, which can be divided into approaches where one Pareto optimal solution is computed online and approaches with an offline phase during which the MOCP is solved (in this article, algorithms where Artificial Neural Networks (ANN) have to be trained beforehand are nonetheless assigned to the first category if the optimization is performed entirely online).

When implementing a multiobjective MPC (MOMPC) algorithm, one should keep in mind that, regardless of the algorithm used, the resulting trajectory need not be Pareto optimal, even if each single step is, cf. [129]. A remedy to this issue is presented in [130], where the selection of compromise solutions is restricted to a part of the Pareto front that is determined in the first MPC step. Due to this, upper bounds for the objective function values can be guaranteed.

\subsection{Online Multiobjective Optimization}

In the classical MPC framework, the optimal control problem is solved online within the sample time $h$. Since it is in general impossible to approximate the entire Pareto set sufficiently accurately within this time frame, there are three alternatives:

1. compute a single Pareto-optimal solution according to some predefined preference,

2. compute only a rough approximation of the Pareto set,

3. compute an arbitrary Pareto-optimal control that satisfies additional constraints (e.g., stability).

In the first approach, the objective function is scalarized using, for instance, the weighted sum method (12) or the reference point method (13). In this situation, well-established approaches from scalar-valued MPC exist on which one can build. First results using the weighted sum method have appeared in [131]. In [132,133], the authors use the same scalarization for an MPC problem with 
convex objective functions. In this situation, it is guaranteed that any Pareto-optimal solution can be computed using weighted sums, and the weights can be adapted online according to a decision maker's preference. Due to the convexity, stability can be proven for the resulting MPC algorithm. In [134], this approach is extended by providing gradient information of the objectives with respect to the weight vector $\alpha$. This way, the weights can be adapted in such a way that a desired change in the objective space is realized. For non-convex problems, the weighted sum method is incapable of computing the entire Pareto set. Therefore, in [135], a variation of the reference point method is applied, where the $\operatorname{target} T$ is the utopian point $J^{*}$, i.e., the vector of the individual minima. This way, also non-convex problems can be treated. In fact, due to the reference point method, the objective function is always convex [109], which can be exploited during the optimization. Alternative scalarization methods are the $\epsilon$-constraint method [136] or lexicographic ordering [137].

A disadvantage of a priori scalarization is that it is often difficult to select the scalarization parameter in such a way that a desired trade-off solution is obtained, and the remedy proposed in [134] is only applicable to a specific class of problems. Therefore, an alternative approach is to quickly compute a rough approximation of the entire Pareto set and then select the desired control online. Such methods have been proposed by many authors. The general approach is to us an MOEA and stop the computation after a few iterations. In the next step, one of these suboptimal solutions is selected. This selection is realized by specifying a weight vector for the objectives in [138-141] and by the satisficing trade-off method in [142].

As a third option, we can compute a single Pareto-optimal point without specifying which one we are specifically interested in as long as it satisfies additional constraints such as the stability of the system. Approaches of this type have been developed in [136,143], where a game theoretic approach is used.

\subsection{Offline-Online Decomposition}

A well-known trick to avoid heavy online computations is to introduce an offline-online decomposition (very similar to meta modeling approaches where surrogate models are constructed before solving the MOCP). This means that the Pareto set is computed beforehand, and in the online phase, an optimal compromise is selected according to a decision maker's preference or some heuristic based on the system state or the environment.

Many of the approaches that fall into this category use a standard feedback controller instead of MPC; see [144] for a short review concerning methods using scalarization and offline PID controller optimization. In the offline phase, a Pareto set is computed for the controller parameters. Possible objectives are, among many others, overshooting behavior, energy efficiency or robustness. Algorithms of this type have been proposed in [145-147] using MOEAs and in [148-150] using set-oriented methods.

An alternative approach is motivated by explicit MPC, i.e., the idea of solving many MOPs offline such that the correct solution can be extracted from a library in the online phase. Such a method has been proposed in [48]. In the offline phase, one has to identify all possible scenarios that can occur in the online phase. Such a scenario consists of both system states, as well as constraints. This results in a large number of MOPs that have to be solved. In order to reduce this number, symmetries in the problem are exploited. To this end, a concept known as motion primitives $[151,152]$ is extended. In short, this means that if:

$$
\arg \min _{u \in \mathcal{U}} \mathrm{MOP}_{1}=\arg \min _{u \in \mathcal{U}} \mathrm{MOP}_{2},
$$

where $\mathrm{MOP}_{1}$ and $\mathrm{MOP}_{2}$ are two problem instances from the offline library, then we only have to solve one of the problems in order to have a Pareto-optimal solution for both. Moreover, if two problem instances vary only slightly, one can use a previously-computed solution as a good initial guess for the next MOCP to further decrease the computational effort [73]. In the online phase, the correct Pareto set is selected from the library (according to the system state and the constraints), and an optimal 
compromise is selected according so a decision maker's preference $\alpha$. In contrast to the affine linear solutions, which can be computed in explicit MPC for linear-quadratic problems, one has to rely on interpolation between solutions in the nonlinear setting.

\subsubsection{Example: Autonomous Driving}

We here want to demonstrate the superiority of multiobjective approaches over scalar-valued MPC using the example of autonomously-driving electric vehicles $[47,48,153]$. The problem there is to find the set of optimal engine torque profiles such that the velocity is maximized while the energy consumption is minimized. Additional constraints have to be taken into account such as speed limits or stop signs. The system dynamics are described by a four-dimensional, highly nonlinear ODE for the vehicle velocity, the battery state of charge and two battery voltage drops, cf. [153] for details. Numerical investigations reveal several symmetries in the system such that the only relevant state for a scenario is the current velocity, whereas all other states only have a minor influence on the solution of the MOP. Consequently, the velocity, as well as the constraints form the above-mentioned scenarios; see Figure 10a for an illustration. For example, a scenario could be that the current velocity is $60 \mathrm{~km} / \mathrm{h}$ and that the speed limit is currently increasing from $50-100 \mathrm{~km} / \mathrm{h}$, cf. Scenario (II) in Figure 10a. We then solve the MOP for this scenario and store the Pareto set in a library. By discretizing the velocity into steps of 0.1 (i.e., $v\left(t_{0}\right)=\ldots, 59.9,60.0,60.1, \ldots$ ), we have to solve 1727 MOPs in total in the offline phase.

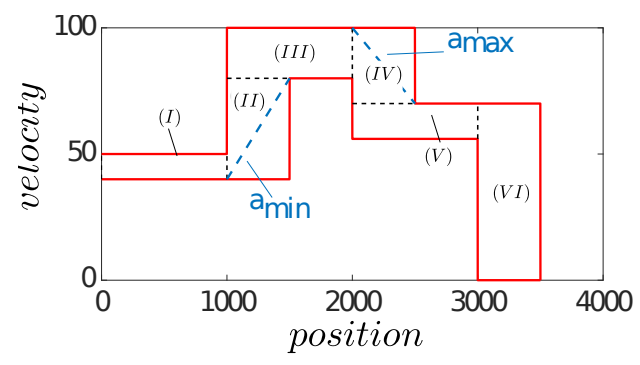

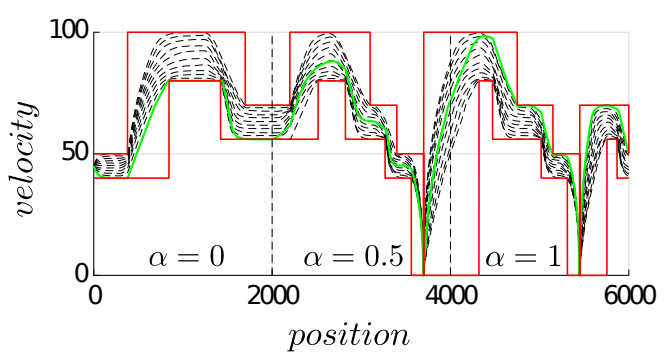

(b) (a)

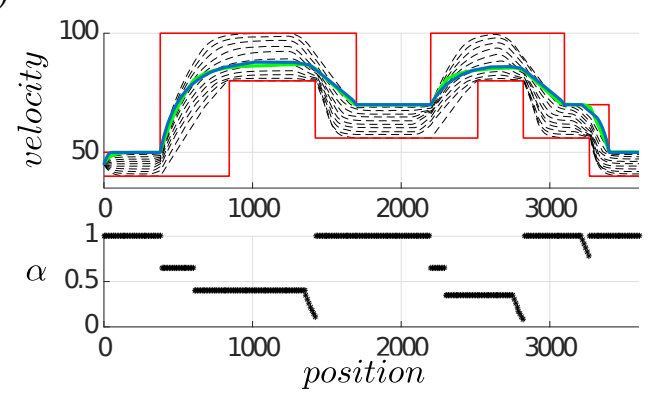

(c)

Figure 10. Results for the offline-online MPC approach from [48]. (a) Different constraint scenarios ((I)-(VI)), i.e., constant velocity, acceleration, deceleration and stopping. (b) Example track driven with the MPC algorithm. The red lines define the velocity bounds; the black dashed lines are trajectories corresponding to a constant weight $\alpha$; and the green line is a trajectory where the weight is changed from 0 (energy efficient) over 0.5 (average) to 1 (fast). (c) Comparison between the MPC algorithm (coupled with a simple heuristic for the weighting) and the global optimum obtained via dynamic programming. 
Table 2. Overview of publications (in chronological order) for multiobjective feedback control.

\begin{tabular}{|c|c|}
\hline \multicolumn{2}{|c|}{ Algorithms without Offline Phase: Computation of Single Points } \\
\hline $\begin{array}{l}\text { Kerrigan et al. [131], } \\
\text { Wojsznis et al. [154] }\end{array}$ & Scalarization via Weighted Sum (WS) \\
\hline $\begin{array}{l}\text { Kerrigan and Maciejowski [155], } \\
\text { He et al. [137] }\end{array}$ & Scalarization via lexicographic ordering \\
\hline $\begin{array}{l}\text { Bemporad and Muñoz } \\
\text { de la Peña }[132,133]\end{array}$ & $\begin{array}{l}\text { Scalarization via WS for convex objectives, guaranteed stability for } \\
\text { large gain vs. noise robust stabilizing objectives }\end{array}$ \\
\hline Geisler and Trächtler [134] & WS, online adaptation of weights using gradient information \\
\hline Maestre et al. [143] & Scalarization via game-theoretic approach \\
\hline $\begin{array}{l}\text { Zavala and Flores- } \\
\text { Tlacuahuac [135] }\end{array}$ & Scalarization via reference point approach \\
\hline Hackl et al. [129] & Scalarization via WS for Linear Time-Invariant (LTI) systems \\
\hline Zavala [136] & Scalarization via $\epsilon$-constraint: economic objective, stability as constraint \\
\hline Grüne and Stieler [130] & Economic objectives, performance bounds via selection criterion \\
\hline \multicolumn{2}{|c|}{ Algorithms without Offline Phase: Approximation of the Entire Pareto Set } \\
\hline $\begin{array}{l}\text { Laabidi et al. [138,140], } \\
\text { Garcìa et al. [141] }\end{array}$ & $\begin{array}{l}\text { ANN for state prediction, optimization via } \\
\text { MOEA, selection of Pareto point via WS }\end{array}$ \\
\hline Bouani et al. [139] & ANN for state prediction, comparison of two MOEAs and WS for MOP \\
\hline Nakayama et al. [142] & Few MOEA iterations online, selection via satisficing trade-off method \\
\hline \multicolumn{2}{|r|}{ Algorithms with Offline Phase } \\
\hline $\begin{array}{l}\text { Fonseca [145], } \\
\text { Herreros et al. [146] }\end{array}$ & $\begin{array}{l}\text { Offline computation of Pareto optimal controller parameters } \\
\text { using MOEA }\end{array}$ \\
\hline Scherer et al. [156] & $\begin{array}{l}\text { Robust control using a common Lyapunov function for multiple } \\
\text { stability criteria }\end{array}$ \\
\hline Ben Aicha et al. [147] & $\begin{array}{l}\text { Offline computation of Pareto optimal controllers parameters via EA } \\
\text { and WS, online selection according to geometric mean of objectives }\end{array}$ \\
\hline Krüger et al. [148] & $\begin{array}{l}\text { Offline computation of Pareto optimal controllers parameters via Set } \\
\text { oriented methods, parametric model reduction for increased efficiency }\end{array}$ \\
\hline $\begin{array}{l}\text { Hernández et al. [149], } \\
\text { Xiong et al. [150] }\end{array}$ & $\begin{array}{l}\text { Offline computation of Pareto-optimal controllers parameters via } \\
\text { simple cell mapping }\end{array}$ \\
\hline Peitz et al. [48] & Offline-online decomposition similar to explicit MPC \\
\hline \multicolumn{2}{|r|}{ Applications } \\
\hline Zambrano and Camacho [157] & MOMPC of a solar refrigeration plant via scalarization \\
\hline Porfírio et al. [158] & MOMPC of an industrial splitter using a min-max reformulation \\
\hline Pedersen and Yang [159] & MO PID controller design for magnetic levitation systems via MOEA \\
\hline Li et al. [160] & Multiobjective adaptive cruise control for vehicles \\
\hline Hu et al. [161] & MOMPC of high-power converters via WS \\
\hline Núñez et al. [162] & MOMPC of dynamic pickup and delivery problems using MOEA \\
\hline Peitz et al. [163] & $\begin{array}{l}\text { MOMPC of an industrial laundry, scalarization of a traveling } \\
\text { salesman problem via WS }\end{array}$ \\
\hline
\end{tabular}

In the online phase, we now select the relevant Pareto set from the library and, according to a decision maker's preference, apply one of the Pareto-optimal controls to the electric vehicle. This is done repeatedly such that a feedback loop is realized. The result is illustrated in Figure $10 \mathrm{~b}$ for an example track, where the black lines correspond to constant weighting of the two criteria and the green 
line corresponds to a varying weight. This way, a flexible cruise control is established where the driver can quickly adapt, for instance, to changing energy requirements.

As has been mentioned before, an alternative to interactively choosing a weight is to implement some heuristic that automatically chooses a weight based on the current situation. Such an approach is visualized in Figure 10c, where the weighting depends on the vehicle velocity, as well as on current and future velocity constraints. For a simpler track, it is possible to compute a globally optimal solution for a scalarized objective using dynamic programming. We see that with the heuristic, the MOMPC approach yields trajectories close to the global optimum while only having finite horizon information.

\subsection{Summary}

We again conclude the section by giving a summary of publications where multiobjective optimization is applied in a real-time context, cf. Table 2. The publications are divided into four categories. The first two contain algorithms where the MOP is solved online. The categories differ in whether a single point is computed or the entire set is approximated. Consequently an offline phase is not required except in the case where surrogate models are trained in order to accelerate the online computations. The third category then contains the methods with an offline optimization phase, and some applications are mentioned in the fourth category.

\section{Reduction Techniques for Many-Objective Optimization Problems}

Another important restricting factor in multiobjective optimization is the number of objectives [164]. For MOPs with four or more objectives, the term Many-Objective Optimization (MaOP) has been coined, and over the past few years, many researchers have dedicated their work to address MaOPs and the issues arising from the curse of dimensionality, cf. [165] for an overview, [166,167] for new concepts for identifying non-dominated solutions and [168-172] for evolutionary approaches.

A popular approach for MaOPs is comprised of interactive methods [173-178]. These methods do not compute the entire set of optimal compromises, but instead interactively explore the Pareto set. Starting at the current Pareto-optimal solution, a decision maker can choose in which direction to proceed, i.e., which objective to improve at the expense of some other, currently less important objective. The approach in [178], for example, allows for Pareto-optimal movements both in the decision and objective space. One of the main advantages of interactive methods is the reduced computational effort, especially in the presence of many criteria, since it is not affected significantly by the dimension of the Pareto set. Moreover, this way, decision making from a vast number of Pareto-optimal solutions is avoided, which can be overwhelming for a decision maker. Consequently, interpretability and usability are increased.

Besides interactive methods, several reduction techniques have been proposed in the context of many-objective optimization, and although it is not the main theme of this review article, we want to give a brief overview of these reduction approaches since they also aim at increasing the efficiency of solving MOPs. These reduction techniques can be divided into two main categories. The first one is objective reduction, where the aim is to reduce the number of objectives while (approximately) preserving the Pareto set. The observation behind this is that not all objectives are of equal importance to the structure of the Pareto set, which is measured by the degree of conflict [179]. Consequently, when it is possible to identify the main contributors to the Pareto set, then one can solve a reduced problem taking into account only these most important objectives. Different approaches have been proposed for this identification step, all of which use a set of sample points. In [179], both exact and inexact algorithms are proposed for selecting a subset of objectives such that only those points in the Pareto set are lost that are worse in all remaining objectives by a constant $\delta$ or more. This approach is also exploited in [180]. In [181,182], POD (cf. Section 3.3) is used to identify such a subset, and a related concept is implemented in [183] using hyperplanes. In [184], an entropy-based approach is presented, and in [185], the relevant subset is selected multiple times within an evolutionary procedure. 
A slightly different approach is pursued in [186], where the authors split large decision spaces into several smaller ones according to the relevance of decision variables for specific objectives.

The method proposed in [187] possesses characteristics of the first category, namely objective reduction, as well as of the second category, which is the exploitation of the structure of Pareto sets and front. Therein, first the corners of the Pareto front are identified, and this information is used to select the relevant objectives. Algorithms of the second type all exploit this hierarchical structure. This means that under certain assumptions, the Pareto front is bounded by the Pareto fronts of subproblems where one or more objectives have been neglected, cf. [188,189]. This way, the solution can be computed by a hierarchical approach where, starting with scalar problems, the boundary is computed before, finally, the interior is obtained. Very recently, results about the hierarchical structure of the Pareto set, i.e., in decision space, have appeared; see $[73,190]$ for details. This approach is illustrated in Figure 11 where the solution to an MOP with four objectives is shown, as well as the Pareto sets of the subproblems with three and two objectives, respectively.

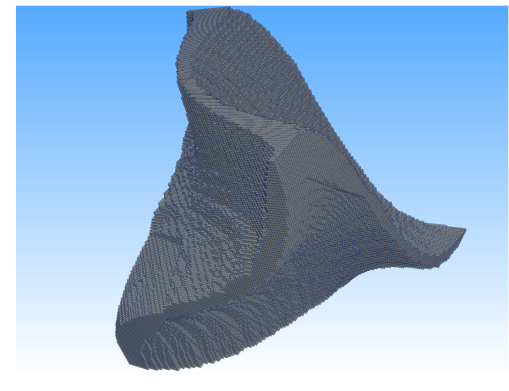

(a)

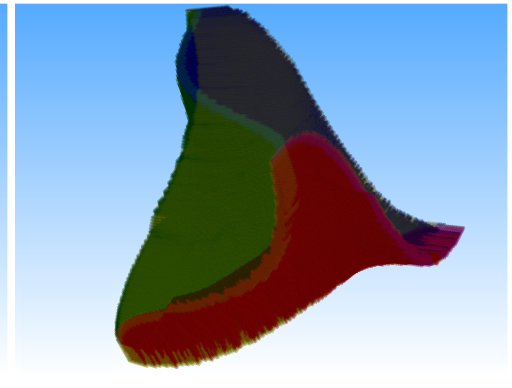

(b)

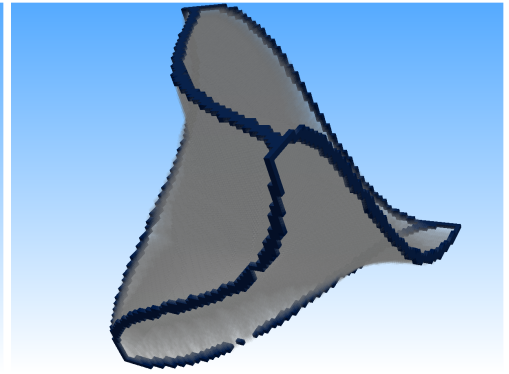

(c)

Figure 11. Visualization of the hierarchical structure of Pareto sets. (a) Pareto set of an example problem with $J: \mathbb{R}^{3} \rightarrow \mathbb{R}^{4}$. (b) The four Pareto sets taking only three objectives into account form the boundary of the original Pareto set. (c) The Pareto sets in (b) are again bounded by the respective bi-objective subproblems.

\section{Future Directions}

This survey has given an overview of recent advances in the context of accelerating multiobjective optimization. These are surrogate models, feedback control and objective reduction techniques. Similar to almost every other field of science, it can be expected that the immense developments in data-based methods will also have a major impact on research in multiobjective optimization, in particular in the context of surrogate modeling. A very large number of researchers from the dynamical systems community is working on data-based methods using the Koopman operator, which is an infinite-dimensional, but linear operator describing the dynamics of observables [191,192]. Significant effort has been put into the development of numerical methods for approximating this operator from data; see, e.g., $[193,194]$. This way, the dynamics of observations can be reconstructed entirely from data and without any knowledge of the underlying system dynamics. In a way, this allows us to merge the two surrogate modeling categories from Sections 3.2 and 3.3 since we can approximate the dynamics not only of the state, but directly of the objectives. Several methods have recently been proposed to use the Koopman operator for data-based controller design, both in simulations [195-198], as well as experiments $[199,200]$. The results are very promising, such that it is just a matter of time until these methods are utilized for multiobjective optimal control.

In the same manner, machine learning techniques [201] will very likely gain more and more attention, both in multiobjective optimization, as well as optimal control. There are already many papers on this topic or related ones, and the number is growing quickly. 
Author Contributions: S.P. is responsible for the literature research and writing large parts of the paper. M.D. directed and supervised the research presented in the various examples and contributed to writing the final manuscript.

Acknowledgments: This work is supported by the Priority Programme SPP 1962 "Non-smooth and Complementarity-based Distributed Parameter Systems" of the German Research Foundation (DFG).

Conflicts of Interest: The authors declare no conflict of interest.

\section{References}

1. Knowles, J.; Nakayama, H. Meta-Modeling in Multiobjective Optimization. In Multiobjective Optimization: Interactive and Evolutionary Approaches; Branke, J., Deb, K., Miettinen, K., Slowinski, R., Eds.; Springer: Berlin/Heidelberg, Germany, 2008; pp. 245-284.

2. Tabatabaei, M.; Hakanen, J.; Hartikainen, M.; Miettinen, K.; Sindhya, K. A survey on handling computationally expensive multiobjective optimization problems using surrogates: Non-nature inspired methods. Struct. Multidiscip. Optim. 2015, 52, 1-25. [CrossRef]

3. Chugh, T.; Sindhya, K.; Hakanen, J.; Miettinen, K. A survey on handling computationally expensive multiobjective optimization problems with evolutionary algorithms. Soft Comput. 2017, 1-30. [CrossRef]

4. Miettinen, K. Nonlinear Multiobjective Optimization; Springer Science and Business Media: Berlin, Germany, 2012.

5. Ehrgott, M. Multicriteria Optimization, 2nd ed.; Springer: Berlin/Heidelberg, Germany; New York, NY, USA, 2005.

6. Hindi, H.A.; Hassibi, B.; Boyd, S.B. Multiobjective $\mathcal{H}_{2} / \mathcal{H}_{\infty}$-Optimal Control via Finite Dimensional $Q$-Parametrization and Linear Matrix Inequalities. In Proceedings of the American Control Conference, Philadelphia, PA, USA, 24-26 June 1998; pp. 3244-3249.

7. Zhu, Q.J. Hamiltonian Necessary Conditions for a Multiobjective Optimal Control Problem with Endpoint Constraints. SIAM J. Control Optim. 2000, 39, 97-112. [CrossRef]

8. Gambier, A.; Badreddin, E. Multi-objective optimal control: An overview. In Proceedings of the 16th IEEE International Conference on Control Applciations, Singapore, 1-3 October 2007; pp. 170-175.

9. Tröltzsch, F. Optimal Control Of Partial Differential Equations. In Graduate Studies in Mathematics; American Mathematical Society: Providence, RI, USA, 2010; Volume 112.

10. Hinze, M.; Pinnau, R.; Ulbrich, M.; Ulbrich, S. Optimization with PDE Constraints; Springer Science+Business Media: Berlin, Germany, 2009.

11. Ober-Blöbaum, S. Discrete Mechanics and Optimal Control. Ph.D. Thesis, University of Paderborn, Paderborn, Germany, 2008.

12. Schilders, W.H.A.; van der Vorst, H.A.; Rommes, J. Model Order Reduction; Springer: Berlin/Heidelberg, Germany, 2008.

13. Karush, W. Minima of Functions of Several Variables with Inequalities as Side Constraints. Master's Thesis, University of Chicago, Chicago, IL, USA, 1939.

14. Kuhn, H.W.; Tucker, A.W. Nonlinear programming. In Proceedings of the 2nd Berkeley Symposium on Mathematical and Statsitical Probability, Oakland, CA, USA, 31 July-12 August 1950; University of California Press: Berkeley, CA, USA, 1951; pp. 481-492.

15. Romaus, C.; Böcker, J.; Witting, K.; Seifried, A.; Znamenshchykov, O. Optimal energy management for a hybrid energy storage system combining batteries and double layer capacitors. In Proceedings of the IEEE Energy Conversion Congress and Exposition, San Jose, CA, USA, 20-24 September 2009; pp. 1640-1647.

16. Hillermeier, C. Nonlinear Multiobjective Optimization: A Generalized Homotopy Approach; Birkhäuser: Basel, Switzerland, 2001.

17. Schütze, O.; Dell'Aere, A.; Dellnitz, M. On Continuation Methods for the Numerical Treatment of Multi-Objective Optimization Problems. Available online: http://drops.dagstuhl.de/opus/volltexte/ 2005/349/pdf/04461.SchuetzeOliver.Paper.349.pdf (accessed on 31 May 2018).

18. Deb, K. Multi-Objective Optimization Using Evolutionary Algorithms; John Wiley and Sons: Hoboken, NJ, USA, 2001; Volume 16.

19. Coello Coello, C.A.; Lamont, G.B.; Van Veldhuizen, D.A. Evolutionary Algorithms for Solving Multi-Objective Problems; Springer Science and Business Media: Berlin, Germany, 2007; Volume 2. 
20. Zitzler, E.; Laumanns, M.; Thiele, L. SPEA2: Improving the Strength Pareto Evolutionary Algorithm. Available online: https:/ / www.research-collection.ethz.ch/bitstream/handle/20.500.11850/145755/eth24689-01.pdf (accessed on 31 May 2018).

21. Deb, K.; Pratap, A.; Agarwal, S.; Meyarivan, T. A Fast and Elitist Multiobjective Genetic Algorithm: NSGA-II. IEEE Trans. Evol. Comput. 2002, 6, 182-197. [CrossRef]

22. Zhou, A.; Qu, B.Y.; Li, H.; Zhao, S.Z.; Suganthan, P.N.; Zhang, Q. Multiobjective evolutionary algorithms: A survey of the state of the art. Swarm Evol. Comput. 2011, 1, 32-49. [CrossRef]

23. Ong, Y.S.; Lim, M.H.; Chen, X. Memetic Computation-Past, Present and Future. IEEE Comput. Intell. Mag. 2010, 5, 24-31. [CrossRef]

24. Neri, F.; Cotta, C.; Moscato, P. Handbook of Memetic Algorithms; Springer: Berlin, Germany, 2012; Volume 379.

25. Schütze, O.; Alvarado, S.; Segura, C.; Landa, R. Gradient subspace approximation: A direct search method for memetic computing. Soft Comput. 2017, 21, 6331-6350. [CrossRef]

26. Schütze, O.; Martín, A.; Lara, A.; Alvarado, S.; Salinas, E.; Coello Coello, C.A. The directed search method for multi-objective memetic algorithms. Comput. Optim. Appl. 2016, 63, 305-332. [CrossRef]

27. Dellnitz, M.; Schütze, O.; Hestermeyer, T. Covering Pareto sets by Multilevel Subdivision Techniques. J. Optim. Theory Appl. 2005, 124, 113-136. [CrossRef]

28. Jahn, J. Multiobjective Search Algorithm with Subdivision Technique. Comput. Optim. Appl. 2006, 35, 161-175. [CrossRef]

29. Schütze, O.; Witting, K.; Ober-Blöbaum, S.; Dellnitz, M. Set Oriented Methods for the Numerical Treatment of Multiobjective Optimization Problems. In EVOLVE-A Bridge between Probability, Set Oriented Numerics and Evolutionary Computation; Tantar, E., Tantar, A.A., Bouvry, P., Del Moral, P., Legrand, P., Coello Coello, C.A., Schütze, O., Eds.; Studies in Computational Intelligence; Springer: Berlin/Heidelberg, Germany, 2013; Volume 447, pp. 187-219.

30. Bosman, P.A.N. On Gradients and Hybrid Evolutionary Algorithms. IEEE Trans. Evol. Comput. 2012, 16, 51-69. [CrossRef]

31. Fliege, J.; Svaiter, B.F. Steepest descent methods for multicriteria optimization. Math. Methods Oper. Res. 2000, 51, 479-494. [CrossRef]

32. Schäffler, S.; Schultz, R.; Weinzierl, K. Stochastic Method for the Solution of Unconstrained Vector Optimization Problems. J. Optim. Theory Appl. 2002, 114, 209-222. [CrossRef]

33. Gebken, B.; Peitz, S.; Dellnitz, M. A Descent Method for Equality and Inequality Constrained Multiobjective Optimization Problems. arXiv 2017, arXiv:1712.03005.

34. Bosman, P.A.N.; de Jong, E.D. Exploiting gradient information in numerical multi-objective evolutionary optimization. In Proceedings of the 2005 Conference on Genetic and Evolutionary Computation, Washington, DC, USA, 25-29 June 2005; pp. 755-762.

35. Fliege, J.; Grana Drummond, L.M.; Svaiter, B.F. Newton's method for multiobjective optimization. SIAM J. Optim. 2009, 20, 602-626. [CrossRef]

36. Fliege, J.; Vaz, A.I.F. A SQP Type Method for Constrained Multiobjective Optimization. Available online: http:/ / www.optimization-online.org/DB_FILE/2015/05/4929.pdf (accessed 31 May 2018).

37. Brown, M.; Smith, R.E. Directed multi-objective optimization. Int. J. Comput. Syst. Signals 2005, 6, 3-17.

38. Harada, K.; Sakuma, J.; Kobayashi, S. Local Search for Multiobjective Function Optimization: Pareto Descent Method. In Proceedings of the 8th Annual Conference on Genetic and Evolutionary Computation (GECCO 06), Seattle, WA, USA, 8-12 July 2006; pp. 659-666.

39. Harada, K.; Sakuma, J.; Ono, I.; Kobayashi, S. Constraint-Handling Method for Multi-objective Function Optimization: Pareto Descent Repair Operator. In Evolutionary Multi-Criterion Optimization; Obayashi, S., Deb, K., Poloni, C., Hiroyasu, T., Murata, T., Eds.; Springer: Berlin/Heidelberg, Germany, 2007; pp. 156-170.

40. Custódio, A.L.; Madeira, J.F.A.; Vaz, A.I.F.; Vicente, L.N. Direct multisearch for multiobjective optimization. SIAM J. Optim. 2011, 21, 1109-1140. [CrossRef]

41. Désidéri, J.A. Mutiple-Gradient Descent Algorithm for Multiobjective Optimization. In Proceedings of the European Congress on Computational Methods in Applied Sciences and Engineering, Vienna, Austria, 10-14 September 2012; pp. 3974-3993.

42. Garduno-Ramirez, R.; Lee, K.Y. Multiobjective optimal power plant operation through coordinate control with pressure set point scheduling. IEEE Trans. Energy Convers. 2001, 16, 115-122. [CrossRef] 
43. Logist, F.; Houska, B.; Diehl, M.; van Impe, J. Robust multi-objective optimal control of uncertain (bio)chemical processes. Chem. Eng. Sci. 2011, 66, 4670-4682. [CrossRef]

44. Ober-Blöbaum, S.; Ringkamp, M.; zum Felde, G. Solving Multiobjective Optimal Control Problems in Space Mission Design using Discrete Mechanics and Reference Point Techniques. In Proceedings of the 51st IEEE International Conference on Decision and Control, Maui, HI, USA, 10-13 December 2012; pp. 5711-5716.

45. Lu, J.; DePoyster, M. Multiobjective optimal suspension control to achieve integrated ride and handling performance. IEEE Trans. Control Syst. Technol. 2002, 10, 807-821.

46. Geisler, J.; Witting, K.; Trächtler, A.; Dellnitz, M. Multiobjective optimization of control trajectories for the guidance of a rail-bound vehicle. IFAC Proc. 2008, 17, 4380-4386. [CrossRef]

47. Dellnitz, M.; Eckstein, J.; Flaßkamp, K.; Friedel, P.; Horenkamp, C.; Köhler, U.; Ober-Blöbaum, S.; Peitz, S.; Tiemeyer, S. Multiobjective Optimal Control Methods for the Development of an Intelligent Cruise Control. In Progress in Industrial Mathematics at ECMI 2014; Russo, G., Capasso, V., Nicosia, G., Romano, V., Eds.; Springer: Berlin, Germany, 2017; pp. 633-641.

48. Peitz, S.; Schäfer, K.; Ober-Blöbaum, S.; Eckstein, J.; Köhler, U.; Dellnitz, M. A Multiobjective MPC Approach for Autonomously Driven Electric Vehicles. IFAC PapersOnLine 2017, 50, 8674-8679. [CrossRef]

49. Brunton, S.L.; Noack, B.R. Closed-Loop Turbulence Control: Progress and Challenges. Appl. Mech. Rev. 2015, 67, 1-48. [CrossRef]

50. Vemuri, V.R.; Cedeno, W. A New Genetic Algorithm for Multi-objective Optimization in Water Resource Management. In Proceedings of the IEEE International Conference on Evolutionary Computation, Perth, Australia, 29 November-1 December 1995; Volume 1, pp. 1-11.

51. Rosehart, W.D.; Cañizares, C.A.; Quintana, V.H. Multiobjective optimal power flows to evaluate voltage security costs in power networks. IEEE Trans. Power Syst. 2003, 18, 578-587. [CrossRef]

52. Lotov, A.V.; Kamenev, G.K.; Berezkin, V.E.; Miettinen, K. Optimal control of cooling process in continuous casting of steel using a visualization-based multi-criteria approach. Appl. Math. Model. 2005, 29, 653-672. [CrossRef]

53. Albunni, M.N.; Rischmuller, V.; Fritzsche, T.; Lohmann, B. Multiobjective Optimization of the Design of Nonlinear Electromagnetic Systems Using Parametric Reduced Order Models. IEEE Trans. Magn. 2009, 45, 1474-1477. [CrossRef]

54. Ober-Blöbaum, S.; Padberg-Gehle, K. Multiobjective optimal control of fluid mixing. Proc. Appl. Math. Mech. 2015, 15, 639-640. [CrossRef]

55. Ramos, A.M.; Glowinski, R.; Periaux, J. Nash Equilibria for the Multiobjective Control of Linear Partial Differential Equations 1. J. Optim. Theory Appl. 2002, 112, 457-498. [CrossRef]

56. Borzì, A.; Kanzow, C. Formulation and Numerical Solution of Nash Equilibrium Multiobjective Elliptic Control Problems. SIAM J. Control Optim. 2013, 51, 718-744. [CrossRef]

57. Triantaphyllou, E. Multi-criteria decision making methods. In Multi-Criteria Decision Making Methods: A Comparative Study; Springer: Berlin, Germany, 2000; pp. 5-21.

58. White, D.J. Epsilon efficiency. J. Optim. Theory Appl. 1986, 49, 319-337. [CrossRef]

59. Hughes, E.J. Evolutionary Multi-objective Ranking with Uncertainty and Noise. In Evolutionary Multi-Criterion Optimization: First International Conference, Zurich, Switzerland, March 7-9; Zitzler, E., Thiele, L., Deb, K., Coello Coello, C.A., Corne, D., Eds.; Springer: Berlin/Heidelberg, Germany, 2001; pp. 329-343.

60. Deb, K.; Mohan, M.; Mishra, S. Evaluating the epsilon-Domination Based Multi-Objective Evolutionary Algorithm for a Quick Computation of Pareto-Optimal Solutions. Evol. Comput. 2005, 13, 501-525. [CrossRef] [PubMed]

61. Basseur, M.; Zitzler, E. A Preliminary Study on Handling Uncertainty in Indicator-Based Multiobjective Optimization. In Workshops on Applications of Evolutionary Computation; Rothlauf, F., Branke, J., Cagnoni, S., Costa, E., Cotta, C., Drechsler, R., Lutton, E., Machado, P., Moore, J.H., Romero, J., et al., Eds.; Springer: Berlin/Heidelberg, Germany, 2006; pp. 727-739.

62. Teich, J. Pareto-Front Exploration with Uncertain Objectives. In Evolutionary Multi-Criterion Optimization; Zitzler, E., Thiele, L., Deb, K., Coello Coello, C.A., Corne, D., Eds.; Springer: Berlin/Heidelberg, Germany, 2001; pp. 314-328.

63. Schütze, O.; Coello Coello, C.A.; Tantar, E.; Talbi, E.G. Computing the Set of Approximate Solutions of an MOP with Stochastic Search Algorithms. In Proceedings of the 10th Annual Conference on Genetic and Evolutionary Computation, Atlanta, GA, USA, 12-16 July 2008; pp. 713-720. 
64. Engau, A.; Wiecek, M.M. Generating $\epsilon$-efficient solutions in multiobjective programming. Eur. J. Oper. Res. 2007, 177, 1566-1579. [CrossRef]

65. Hernández, C.; Sun, J.Q.; Schütze, O. Computing the Set of Approximate Solutions of a Multi-objective Optimization Problem by Means of Cell Mapping Techniques. In EVOLVE-A Bridge between Probability, Set Oriented Numerics, and Evolutionary Computation IV: International Conference held at Leiden University, July 10-13, 2013; Emmerich, M., Deutz, A., Schütze, O., Bäck, T., Tantar, E., Tantar, A.A., Moral, P.D., Legrand, P., Bouvry, P., Coello, C.A., Eds.; Springer International Publishing: Basel, Switzerland, 2013; pp. 171-188.

66. Peitz, S.; Dellnitz, M. Gradient-based multiobjective optimization with uncertainties. In NEO 2016; Maldonado, Y., Trujillo, L., Schütze, O., Riccardi, A., Vasile, M., Eds.; Springer: Berlin, Germany, 2018; Volume 731, pp. 159-182.

67. Farina, M.; Amato, P. A fuzzy definition of "optimality" for many-criteria optimization problems. IEEE Trans. Syst. Man Cybern. Part A Syst. Hum. 2004, 34, 315-326. [CrossRef]

68. Singh, A.; Minsker, B.S. Uncertainty-based multiobjective optimization of groundwater remediation design. Water Resour. Res. 2008, 44. [CrossRef]

69. Schütze, O.; Vasile, M.; Coello Coello, C.A. Computing the set of epsilon-efficient solutions in multi-objective space mission design. J. Aerosp. Comput. Inf. Commun. 2009, 8, 53-70. [CrossRef]

70. Deb, K.; Gupta, H.V. Searching for Robust Pareto-optimal Solutions in Multi-objective Optimization. In Evolutionary Multi-Criterion Optimization; Coello Coello, C.A., Hernández Aguirre, A., Zitzler, E., Eds.; Springer: Berlin/Heidelberg, Germany, 2005; pp. 150-164.

71. Xue, Y.; Li, D.; Shan, W.; Wang, C. Multi-objective Robust Optimization Using Probabilistic Indices. In Proceedings of the Third International Conference on Natural Computation, Haikou, China, 24-27 August 2007; Volume 4, pp. 466-470.

72. Dellnitz, M.; Witting, K. Computation of robust Pareto points. Int. J. Comput. Sci. Math. 2009, 2, $243-266$. [CrossRef]

73. Peitz, S. Exploiting Structure in Multiobjective Optimization and Optimal Control. Ph.D. Thesis, Paderborn University, Paderborn, Germany, 2017.

74. Voutchkov, I.; Keane, A.J. Multiobjective optimization using surrogates. In Proceedings of the Adaptive Computing in Design and Manufacture, Bristol, UK, 25-27 April 2006; pp. 167-175.

75. Jin, Y. Surrogate-assisted evolutionary computation: Recent advances and future challenges. Swarm Evol. Comput. 2011, 1, 61-70. [CrossRef]

76. Fang, H.; Rais-Rohani, M.; Liu, Z.; Horstemeyer, M.F. A comparative study of metamodeling methods for multiobjective crashworthiness optimization. Comput. Struct. 2005, 83, 2121-2136. [CrossRef]

77. Sacks, J.; Welch, W.J.; Mitchell, T.J.; Wynn, H.P. Design and Analysis of Computer Experiments. Statist. Sci. 1989, 4, 409-435. [CrossRef]

78. Atkinson, A.C.; Donev, A.N.; Tobias, R.D. Optimum Experimental Designs, With SAS; Oxford University Press: Oxford, UK, 2007.

79. Telen, D.; Logist, F.; Van Derlinden, E.; Tack, I.; Van Impe, J. Optimal experiment design for dynamic bioprocesses: A multi-objective approach. Chem. Eng. Sci. 2012, 78, 82-97. [CrossRef]

80. Ma, C.; Qu, L. Multiobjective Optimization of Switched Reluctance Motors Based on Design of Experiments and Particle Swarm Optimization. IEEE Trans. Energy Convers. 2015, 30, 1144-1153. [CrossRef]

81. Cohn, D.A.; Ghahramani, Z.; Jordan, M.I. Active learning with statistical models. J. Artif. Intell. Res. 1996, 4, 129-145.

82. Yu, K.; Bi, J.; Tresp, V. Active Learning via Transductive Experimental Design. In Proceedings of the 23rd International Conference on Machine Learning, Pittsburgh, PA, USA, 25-29 June 2006; pp. 1081-1088.

83. Benner, P.; Gugercin, S.; Willcox, K. A Survey of Projection-Based Model Reduction Methods for Parametric Dynamical Systems. SIAM Rev. 2015, 57, 483-531. [CrossRef]

84. Peherstorfer, B.; Willcox, K.; Gunzburger, M.D. Survey of Multifidelity Methods in Uncertainty Propagation, Inference, and Optimization. In ACDL Technical Report TR16-1; Massachusetts Institute of Technology: Cambridge, MA, USA, 2016; pp. 1-57.

85. Taira, K.; Brunton, S.L.; Dawson, S.T.M.; Rowley, C.W.; Colonius, T.; McKeon, B.J.; Schmidt, O.T.; Gordeyev, S.; Theofilis, V.; Ukeiley, L.S. Modal Analysis of Fluid Flows: An Overview. AIAA J. 2017, 55, 4013-4041. [CrossRef] 
86. Brenner, S.L.; Scott, R. The Mathematical Theory of Finite Element Methods, 3rd ed.; Springer Science and Business Media: Berlin, Germany, 2003.

87. Volkwein, S. Model reduction using proper orthogonal decomposition. In Lecture Notes; University of Konstanz: Konstanz, Germany, 2011; pp. 1-43.

88. Sirovich, L. Turbulence and the dynamics of coherent structures part I: Coherent structures. Q. Appl. Math. 1987, XLV, 561-571. [CrossRef]

89. Kunisch, K.; Volkwein, S. Control of the Burgers Equation by a Reduced-Order Approach Using Proper Orthogonal Decomposition. J. Optim. Theory Appl. 1999, 102, 345-371. [CrossRef]

90. Kunisch, K.; Volkwein, S. Galerkin proper orthogonal decomposition methods for a general equation in fluid dynamics. SIAM J. Numer. Anal. 2002, 40, 492-515. [CrossRef]

91. Hinze, M.; Volkwein, S. Proper Orthogonal Decomposition Surrogate Models for Nonlinear Dynamical Systems: Error Estimates and Suboptimal Control. In Reduction of Large-Scale Systems; Benner, P., Sorensen, D.C., Mehrmann, V., Eds.; Springer: Berlin/Heidelberg, Germany, 2005; Volume 45, pp. 261-306.

92. Tröltzsch, F.; Volkwein, S. POD a-posteriori error estimates for linear-quadratic optimal control problems. Comput. Optim. Appl. 2009, 44, 83-115. [CrossRef]

93. Lass, O.; Volkwein, S. Adaptive POD basis computation for parametrized nonlinear systems using optimal snapshot location. Comput. Optim. Appl. 2014, 58, 645-677. [CrossRef]

94. Grepl, M.A.; Patera, A.T. A posteriori error bounds for reduced-basis approximations of parametrized parabolic partial differential equations. ESAIM Math. Model. Numer. Anal. 2005, 39, 157-181. [CrossRef]

95. Veroy, K.; Patera, A.T. Certified real-time solution of the parametrized steady incompressible Navier-Stokes equations: Rigorous reduced-basis a posteriori error bounds. Int. J. Numer. Methods Fluids 2005, 47, 773-788. [CrossRef]

96. Haasdonk, B.; Ohlberger, M. Reduced Basis Method for Finite Volume Approximations of Parametrized Evolution Equations. ESAIM Math. Model. Numer. Anal. 2008, 42, 277-302. [CrossRef]

97. Rozza, G.; Huynh, D.B.P.; Patera, A.T. Reduced Basis Approximation and a Posteriori Error Estimation for Affinely Parametrized Elliptic Coercive Partial Differential Equations. Arch. Comput. Methods Eng. 2008, 15, 229-275. [CrossRef]

98. Willcox, K.; Peraire, J. Balanced Model Reduction via the Proper Orthogonal Decomposition. AIAA J. 2002, 40, 2323-2330. [CrossRef]

99. Rowley, C.W. Model Reduction for Fluids, Using Balanced Proper Orthogonal Decomposition. Int. J. Bifurc. Chaos 2005, 15, 997-1013. [CrossRef]

100. Noack, B.R.; Papas, P.; Monkewitz, P.A. The need for a pressure-term representation in empirical Galerkin models of incompressible shear flows. J. Fluid Mech. 2005, 523, 339-365. [CrossRef]

101. Cordier, L.; Abou El Majd, B.; Favier, J. Calibration of POD reduced-order models using Tikhonov regularization. Int. J. Numer. Methods Fluids 2009, 63, 269-296. [CrossRef]

102. Bergmann, M.; Cordier, L.; Brancher, J.P. Optimal rotary control of the cylinder wake using proper orthogonal decomposition reduced-order model. Phys. Fluids 2005, 17, 1-21. [CrossRef]

103. Fahl, M. Trust-region Methods for Flow Control based on Reduced Order Modelling. Ph.D. Thesis, University of Trier, Trier, Germany, 2000.

104. Bergmann, M.; Cordier, L.; Brancher, J.P. Drag Minimization of the Cylinder Wake by Trust-Region Proper Orthogonal Decomposition. In Active Flow Control; King, R., Ed.; Springer: Berlin, Germany, 2007; pp. 309-324.

105. Iapichino, L.; Trenz, S.; Volkwein, S. Multiobjective optimal control of semilinear parabolic problems using POD. In Numerical Mathematics and Advanced Applications (ENUMATH 2015); Karasözen, B., Manguoglu, M., Tezer-Sezgin, M., Goktepe, S., Ugur, Ö., Eds.; Springer: Berlin, Germany, 2016; pp. 389-397.

106. Iapichino, L.; Ulbrich, S.; Volkwein, S. Multiobjective PDE-Constrained Optimization Using the Reduced-Basis Method. Adv. Comput. Math. 2017, 43, 945-972. [CrossRef]

107. Peitz, S.; Ober-Blöbaum, S.; Dellnitz, M. Multiobjective Optimal Control Methods for Fluid Flow Using Model Order Reduction. arXiv 2015, arXiv:1510.05819.

108. Banholzer, S.; Beermann, D.; Volkwein, S. POD-Based Bicriterial Optimal Control by the Reference Point Method. IFAC-PapersOnLine 2016, 49, 210-215. [CrossRef]

109. Banholzer, S.; Beermann, D.; Volkwein, S. POD-Based Error Control for Reduced-Order Bicriterial PDE-Constrained Optimization. Annu. Rev. Control 2017, 44, 226-237. [CrossRef] 
110. Beermann, D.; Dellnitz, M.; Peitz, S.; Volkwein, S. Set-Oriented Multiobjective Optimal Control of PDEs using Proper Orthogonal Decomposition. In Reduced-Order Modeling (ROM) for Simulation and Optimization; Springer: Berlin, Germany, 2018; pp. 47-72.

111. Beermann, D.; Dellnitz, M.; Peitz, S.; Volkwein, S. POD-based multiobjective optimal control of PDEs with non-smooth objectives. Proc. Appl. Math. Mech. 2017, 17, 51-54. [CrossRef]

112. Ong, Y.S.; Nair, P.B.; Keane, A.J. Evolutionary Optimization of Computationally Expensive Problems via Surrogate Modeling. AIAA J. 2003, 41, 687-696. [CrossRef]

113. Ray, T.; Isaacs, A.; Smith, W. Surrogate Assisted Evolutionary Algorithm for Multi-Objective Optimization. In Multi-Objective Optimization; World Scientific: Singapore, 2011; pp. 131-151.

114. Chung, H.S.; Alonso, J. Mutiobjective Optimization Using Approximation Model-Based Genetic Algorithms. In Proceedings of the 10th AIAA/ISSMO Multidisciplinary Analysis and Optimization Conference, Albany, NY, USA, 30 August-1 September 2004.

115. Keane, A.J. Statistical Improvement Criteria for Use in Multiobjective Design Optimization. AIAA J. 2006, 44, 879-891. [CrossRef]

116. Karakasis, M.K.; Giannakoglou, K.C. Metamodel-Assisted Multi-Objective Evolutionary Optimization. In Proceedings of the Sixth Conference on Evolutionary and Deterministic Methods for Design, Optimization and Control with Applications to Industrial and Societal Problems, Munich, Germany, 12-14 September 2005.

117. Knowles, J. ParEGO: A hybrid algorithm with on-line landscape approximation for expensive multiobjective optimization problems. IEEE Trans. Evol. Comput. 2006, 10, 50-66. [CrossRef]

118. Zhang, Q.; Liu, W.; Tsang, E.; Virginas, B. Expensive Multiobjective Optimization by MOEA/D with Gaussian Process Model. IEEE Trans. Evol. Comput. 2010, 14, 456-474. [CrossRef]

119. Chugh, T.; Jin, Y.; Miettinen, K.; Hakanen, J.; Sindhya, K. A Surrogate-Assisted Reference Vector Guided Evolutionary Algorithm for Computationally Expensive Many-Objective Optimization. IEEE Trans. Evol. Comput. 2018, 22, 129-142. [CrossRef]

120. Shimoyama, K.; Jeong, S.; Obayashi, S. Kriging-Surrogate-Based Optimization Considering Expected Hypervolume Improvement in Non-Constrained Many-Objective Test Problems. In Proceedings of the 2013 IEEE Congress on Evolutionary Computation, Cancun, Mexico, 20-23 June 2013; pp. 658-665.

121. Pan, L.; He, C.; Tian, Y.; Wang, H.; Zhang, X.; Jin, Y. A Classification Based Surrogate-Assisted Evolutionary Algorithm for Expensive Many-Objective Optimization. IEEE Trans. Evol. Comput. 2018. [CrossRef]

122. Wang, H.; Jin, Y.; Jansen, J.O. Data-Driven Surrogate-Assisted Multiobjective Evolutionary Optimization of a Trauma System. IEEE Trans. Evol. Comput. 2016, 20, 939-952. [CrossRef]

123. Logist, F.; Houska, B.; Diehl, M.; van Impe, J. Fast Pareto set generation for nonlinear optimal control problems with multiple objectives. Struct. Multidiscip. Optim. 2010, 42, 591-603. [CrossRef]

124. Allgöwer, F.; Zheng, A. Nonlinear Model Predictive Control; Birkhäuser: Basel, Switzerland, 2012; Volume 26.

125. Grüne, L.; Pannek, J. Nonlinear Model Predictive Control, 2rd ed.; Springer International Publishing: Basel, Switzerland, 2017.

126. Rawlings, J.B.; Amrit, R. Optimizing process economic performance using model predictive control. In Nonlinear Model Predictive Control; Springer: Berlin/Heidelberg, Germany, 2009; pp. 119-138.

127. Grüne, L.; Müller, M.A.; Faulwasser, T. Economic Nonlinear Model Predictive Control. Found. Trends Syst. Control 2018, 5, 1-98.

128. Alessio, A.; Bemporad, A. A survey on explicit model predictive control. In Nonlinear Model Predictive Control: Towards New Challenging Applications; Magni, L., Raimondo, D.M., Allgöwer, F., Eds.; Springer: Berlin/Heidelberg, Germany, 2009; Volume 384, pp. 345-369.

129. Hackl, C.M.; Larcher, F.; Dötlinger, A.; Kennel, R.M. Is multiple-objective model-predictive control "optimal”? In Proceedings of the IEEE International Symposium on Sensorless Control for Electrical Drives and Predictive Control of Electrical Drives and Power Electronics (SLED/PRECEDE), München, Germany, 17-19 October 2013; pp. 1-8.

130. Grüne, L.; Stieler, M. Performance Guarantees for Multiobjective Model Predictive Control. Universität Bayreuth, 2017. Available online: https: / / epub.uni-bayreuth.de/3359/ (accessed on 31 May 2018).

131. Kerrigan, E.C.; Bemporad, A.; Mignone, D.; Morari, M.; Maciejowski, J.M. Multi-objective Prioritisation and Reconfiguration for the Control of Constrained Hybrid Systems. In Proceedings of the American Control Conference, Chicago, Illinois, IL, USA, 28-30 June 2000; pp. 1694-1698. 
132. Bemporad, A.; Muñoz de la Peña, D. Multiobjective model predictive control. Automatica 2009, 45, $2823-2830$. [CrossRef]

133. Bemporad, A.; Muñoz de la Peña, D. Multiobjective Model Predictive Control Based on Convex Piecewise Affine Costs. In Proceedings of the European Control Conference, Budapest, Hungary, 23-26 August 2009; pp. 2402-2407.

134. Geisler, J.; Trächtler, A. Control of the Pareto optimality of systems with unknown disturbances. In Proceedings of the IEEE International Conference on Control and Automation, Christchurch, New Zealand, 9-11 December 2009; pp. 695-700.

135. Zavala, V.M.; Flores-Tlacuahuac, A. Stability of multiobjective predictive control: A utopia-tracking approach. Automatica 2012, 48, 2627-2632. [CrossRef]

136. Zavala, V.M. A Multiobjective Optimization Perspective on the Stability of Economic MPC. IFAC-PapersOnLine 2015, 48, 974-980. [CrossRef]

137. He, D.; Wang, L.; Sun, J. On stability of multiobjective NMPC with objective prioritization. Automatica 2015, 57, 189-198. [CrossRef]

138. Laabidi, K.; Bouani, F. Genetic algorithms for multiobjective predictive control. In Proceedings of the First International Symposium on Control, Communications and Signal Processing, Hammamet, Tunisia, 21-24 March 2004; pp. 149-152.

139. Bouani, F.; Laabidi, K.; Ksouri, M. Constrained Nonlinear Multi-objective Predictive Control. In Proceedings of the IMACS Multiconference on Computational Engineering in Systems Applications, Beijing, China, 4-6 October 2006; pp. 1558-1565.

140. Laabidi, K.; Bouani, F.; Ksouri, M. Multi-criteria optimization in nonlinear predictive control. Math. Comput. Simul. 2008, 76, 363-374. [CrossRef]

141. García, J.J.V.; Garay, V.G.; Gordo, E.I.; Fano, F.A.; Sukia, M.L. Intelligent Multi-Objective Nonlinear Model Predictive Control (iMO-NMPC): Towards the 'on-line' optimization of highly complex control problems. Expert Syst. Appl. 2012, 39, 6527-6540. [CrossRef]

142. Nakayama, H.; Yun, Y.; Shirakawa, M. Multi-objective Model Predictive Control. In Multiple Criteria Decision Making for Sustainable Energy and Transportation Systems; Ehrgott, M., Naujoks, B., Stewart, T.J., Wallenius, J., Eds.; Springer: Berlin/Heidelberg, Germany, 2010; pp. 277-287.

143. Maester, J.M.; Muñoz de la Peña, D.; Camacho, E.F. Distributed model predictive control based on a cooperative game. Optim. Control Appl. Methods 2011, 32, 153-176. [CrossRef]

144. Gambier, A. MPC and PID control based on Multi-Objective Optimization. In Proceedings of the American Control Conference, Seattle, WA, USA, 11-13 June 2008; pp. 4727-4732.

145. Fonseca, C.M.M. Multiobjective Genetic Algorithms with Application to Control Engineering Problems. Ph.D. Thesis, University of Sheffield, Sheffield, UK, 1995.

146. Herreros, A.; Baeyens, E.; Perán, J.R. MRCD: A genetic algorithm for multiobjective robust control design. Eng. Appl. Artif. Intell. 2002, 15, 285-301. [CrossRef]

147. Ben Aicha, F.; Bouani, F.; Ksouri, M. Automatic Tuning of GPC synthesis parameters based on Multi-Objective Optimization. In Proceedings of the XIth International Workshop on Symbolic and Numerical Methods, Modeling and Applications to Circuit Design, Gammarth, Tunisia, 5-6 October 2010; pp. 1-5.

148. Krüger, M.; Witting, K.; Trächtler, A.; Dellnitz, M. Parametric Model-Order Reduction in Hierarchical Multiobjective Optimization of Mechatronic Systems. In Proceedings of the 18th IFAC World Congress 2011, Milan, Italy, 28 August-2 September 2011; Elsevier: Oxford, UK, 2011; Volume 18, pp. 12611-12619.

149. Hernández, C.; Naranjani, Y.; Sardahi, Y.; Liang, W.; Schütze, O.; Sun, J.Q. Simple cell mapping method for multi-objective optimal feedback control design. Int. J. Dyn. Control 2013, 1, 231-238. [CrossRef]

150. Xiong, F.R.; Qin, Z.C.; Xue, Y.; Schütze, O.; Ding, Q.; Sun, J.Q. Multi-objective optimal design of feedback controls for dynamical systems with hybrid simple cell mapping algorithm. Commun. Nonlinear Sci. Numer. Simul. 2014, 19, 1465-1473. [CrossRef]

151. Kobilarov, M. Discrete Geometric Motion Control of Autonomous Vehicles. Ph.D. Thesis, University of Southern California, Los Angeles, CA, USA, 2008.

152. Flaßkamp, K.; Ober-Blöbaum, S.; Kobilarov, M. Solving optimal control problems by using inherent dynamical properties. Proc. Appl. Math. Mech. 2010, 10, 577-578. [CrossRef] 
153. Eckstein, J.; Peitz, S.; Schäfer, K.; Friedel, P.; Köhler, U.; Hessel-von Molo, M.; Ober-Blöbaum, S.; Dellnitz, M. A Comparison of two Predictive Approaches to Control the Longitudinal Dynamics of Electric Vehicles. Procedia Technol. 2016, 26, 465-472. [CrossRef]

154. Wojsznis, W.; Mehta, A.; Wojsznis, P.; Thiele, D.; Blevins, T. Multi-objective optimization for model predictive control. ISA Trans. 2007, 46, 351-361. [CrossRef] [PubMed]

155. Kerrigan, E.C.; Maciejowski, J.M. Designing model predictive controllers with prioritised constraints and objectives. In Proceedings of the IEEE International Symposium on Computer Aided Control System Design, Glasgow, UK, 20 September 2002, pp. 33-38.

156. Scherer, C.; Gahinet, P.; Chilali, M. Multiobjective Output-Feedback Control via LMI Optimization. IEEE Trans. Autom. Control 1997, 42, 896-911. [CrossRef]

157. Zambrano, D.; Camacho, E.F. Application of MPC with multiple objective for a solar refrigeration plant. In Proceedings of the IEEE International Conference on Control applications, Glasgow, UK, 18-20 September 2002; pp. 1230-1235.

158. Porfírio, C.R.; Almeida Neto, E.; Odloak, D. Multi-model predictive control of an industrial C3/C4 splitter. Control Eng. Pract. 2003, 11, 765-779. [CrossRef]

159. Pedersen, G.K.M.; Yang, Z. Multi-objective PID-controller tuning for a magnetic levitation system using NSGA-II. In Proceedings of the 8th Annual Conference on Genetic and Evolutionary Computation, Seattle, WA, USA, 8-12 July 2006; pp. 1737-1744.

160. Li, S.; Li, K.; Rajamani, R.; Wang, J. Model Predictive Multi-Objective Vehicular Adaptive Cruise Control. IEEE Trans. Control Syst. Technol. 2011, 19, 556-566. [CrossRef]

161. Hu, J.; Zhu, J.; Lei, G.; Platt, G.; Dorrell, D.G. Multi-objective model-predictive control for high-power converters. IEEE Trans. Energy Convers. 2013, 28, 652-663.

162. Núñez, A.; Cortés, C.E.; Sáez, D.; De Schutter, B.; Gendreau, M. Multiobjective model predictive control for dynamic pickup and delivery problems. Control Eng. Pract. 2014, 32, 73-86. [CrossRef]

163. Peitz, S.; Gräler, M.; Henke, C.; Hessel-von Molo, M.; Dellnitz, M.; Trächtler, A. Multiobjective Model Predictive Control of an Industrial Laundry. Procedia Technol. 2016, 26, 483-490. [CrossRef]

164. Schütze, O.; Lara, A.; Coello Coello, C.A. On the influence of the Number of Objectives on the Hardness of a Multiobjective Optimization Problem. IEEE Trans. Evol. Comput. 2011, 15, 444-455. [CrossRef]

165. Fleming, P.J.; Purshouse, R.C.; Lygoe, R.J. Many-Objective Optimization: An Engineering Design Perspective. In Proceedings of the International Conference on Evolutionary Multi-Criterion Optimization, Guanajuato, Mexico, 9-11 March 2005; pp. 14-32.

166. Kukkonen, S.; Lampinen, J. Ranking-Dominance and Many-Objective Optimization. In Proceedings of the IEEE Congress on Evolutionary Computation, Singapore, 25-28 September 2007; pp. 3983-3990.

167. Bader, J.; Zitzler, E. HypE : An Algorithm for Fast Hypervolume-Based Many-Objective Optimization. Evol. Comput. 2011, 19, 45-76. [CrossRef] [PubMed]

168. Purshouse, R.C.; Fleming, P.J. On the Evolutionary Optimization of Many Conflicting Objectives. IEEE Trans. Evol. Comput. 2007, 11, 770-784. [CrossRef]

169. Ishibuchi, H.; Tsukamoto, N.; Nojima, Y. Evolutionary Many-Objective Optimization: A short Review. In Proceedings of the 2008 IEEE Congress on Evolutionary Computation, Hong Kong, China, 1-6 June 2008; pp. 2419-2426.

170. Von Lücken, C.; Barán, B.; Brizuela, C. A survey on multi-objective evolutionary algorithms for many-objective problems. Comput. Optim. Appl. 2014, 58, 707-756. [CrossRef]

171. Yang, S.; Li, M.; Liu, X.; Zheng, J. A Grid-Based Evolutionary Algorithm for Many-Objective Optimization. IEEE Trans. Evol. Comput. 2013, 17, 721-736. [CrossRef]

172. Li, B.; Li, J.; Tang, K.; Yao, X. Many-Objective Evolutionary Algorithms: A Survey. ACM Comput. Surv. (CSUR) 2015, 48, 13. [CrossRef]

173. Alves, M.J.; Clímaco, J. A review of interactive methods for multiobjective integer and mixed-integer programming. Eur. J. Oper. Res. 2007, 180, 99-115. [CrossRef]

174. Monz, M.; Küfer, K.H.; Bortfeld, T.R.; Thieke, C. Pareto navigation-Algorithmic foundation of interactive multi-criteria IMRT planning. Phys. Med. Biol. 2008, 53, 985-998. [CrossRef] [PubMed]

175. Eskelinen, P.; Miettinen, K.; Klamroth, K.; Hakanen, J. Pareto navigator for interactive nonlinear multiobjective optimization. OR Spectr. 2010, 32, 211-227. [CrossRef] 
176. Cuate, O.; Lara, A.; Schütze, O. A Local Exploration Tool for Linear Many Objective Optimization Problems. In Proceedings of the 13th International Conference on Electrical Engineering, Computing Science and Automatic Control, Mexico City, Mexico, 26-30 September 2016.

177. Cuate, O.; Derbel, B.; Liefooghe, A.; Talbi, E.G. An Approach for the Local Exploration of Discrete Many Objective Optimization Problems. In Proceedings of the 9th International Conference Evolutionary Multi-Criterion Optimization, Münster, Germany, 19-22 March 2017; Trautmann, H., Rudolph, G., Klamroth, K., Schütze, O., Wiecek, M., Jin, Y., Grimme, C., Eds.; Springer International Publishing: Basel, Switzerland, 2017; pp. 135-150.

178. Martin, A.; Schütze, O. Pareto Tracer: A predictor-corrector method for multi-objective optimization problems. Eng. Optim. 2018, 50, 516-536. [CrossRef]

179. Brockhoff, D.; Zitzler, E. Objective Reduction in Evolutionary Multiobjective Optimization: Theory and Applications. Evol. Comput. 2009, 17, 135-166. [CrossRef] [PubMed]

180. Gu, F.; Liu, H.L.; Cheung, Y.M. A Fast Objective Reduction Algorithm Based on Dominance Structure for Many Objective Optimization. In Simulated Evolution and Learning; Shi, Y., Tan, K.C., Zhang, M., Tang, K., Li, X., Zhang, Q., Tan, Y., Middendorf, M., Jin, Y., Eds.; Springer International Publishing: Basel, Switzerland, 2017; pp. 260-271.

181. Deb, K.; Saxena, D.K. On Finding Pareto-Optimal Solutions Through Dimensionality Reduction for Certain Large-Dimensional Multi-Objective Optimization Problems. Kangal Report 2005. Available online: http: / / citeseerx.ist.psu.edu/viewdoc/summary?doi=10.1.1.461.3039 (accessed on 31 May 2018).

182. Saxena, D.K.; Duro, J.A.; Tiwari, A.; Deb, K.; Zhang, Q. Objective reduction in many-objective optimization: Linear and nonlinear algorithms. IEEE Trans. Evol. Comput. 2013, 17, 77-99. [CrossRef]

183. Li, Y.; Liu, H.; Gu, F. An objective reduction algorithm based on hyperplane approximation for many-objective optimization problems. In Proceedings of the 2016 IEEE Congress on Evolutionary Computation, Vancouver, BC, Canada, 24-29 July 2016; pp. 2470-2476.

184. Wang, H.; Yao, X. Objective reduction based on nonlinear correlation information entropy. Soft Comput. 2016, 20, 2393-2407. [CrossRef]

185. Bandyopadhyay, S.; Mukherjee, A. An Algorithm for Many-Objective Optimization With Reduced Objective Computations: A Study in Differential Evolution. IEEE Trans. Evol. Comput. 2015, 19, 400-413. [CrossRef]

186. Wang, H.; Jiao, L.; Shang, R.; He, S.; Liu, F. A Memetic Optimization Strategy Based on Dimension Reduction in Decision Space. Evol. Comput. 2010, 23, 69-100. [CrossRef] [PubMed]

187. Singh, H.K.; Isaacs, A.; Ray, T. A Pareto Corner Search Evolutionary Algorithm and Dimensionality Reduction in Many-Objective Optimization Problems. IEEE Trans. Evol. Comput. 2011, 15, 539-556. [CrossRef]

188. Mueller-Gritschneder, D.; Graeb, H.; Schlichtmann, U. A successive approach to compute the bounded pareto front of practical multiobjective optimization problems. SIAM J. Optim. 2009, 20, 915-934. [CrossRef]

189. Motta, R.D.S.; Afonso, S.M.B.; Lyra, P.R.M. A modified NBI and NC method for the solution of $\mathrm{N}$-multiobjective optimization problems. Struct. Multidiscip. Optim. 2012, 46, 239-259. [CrossRef]

190. Gebken, B.; Peitz, S.; Dellnitz, M. On the hierarchical structure of Pareto critical sets. arXiv 2018, arXiv:1803.06864.

191. Budišić, M.; Mohr, R.; Mezić, I. Applied Koopmanism. Chaos 2012, 22, 047510. [CrossRef] [PubMed]

192. Rowley, C.W.; Mezić, I.; Bagheri, S.; Schlatter, P.; Henningson, D.S. Spectral analysis of nonlinear flows. J. Fluid Mech. 2009, 641, 115-127. [CrossRef]

193. Schmid, P.J. Dynamic mode decomposition of numerical and experimental data. J. Fluid Mech. 2010, 656, 5-28. [CrossRef]

194. Tu, J.H.; Rowley, C.W.; Luchtenburg, D.M.; Brunton, S.L.; Kutz, J.N. On Dynamic Mode Decomposition: Theory and Applications. J. Comput. Dyn. 2014, 1, 391-421.

195. Proctor, J.L.; Brunton, S.L.; Kutz, J.N. Dynamic mode decomposition with control. SIAM J. Appl. Dyn. Syst. 2015, 15, 142-161. [CrossRef]

196. Korda, M.; Mezić, I. Linear predictors for nonlinear dynamical systems: Koopman operator meets model predictive control. arXiv 2016, arXiv:1611.03537.

197. Peitz, S.; Klus, S. Koopman operator-based model reduction for switched-system control of PDEs. arXiv 2017, arXiv:1710:06759. 
198. Peitz, S. Controlling nonlinear PDEs using low-dimensional bilinear approximations obtained from data. arXiv 2018, arXiv:1801.06419.

199. Abraham, I.; De La Torre, G.; Murphey, T.D. Model-Based Control Using Koopman Operators. arXiv 2017, arXiv:1709.01568.

200. Hanke, S.; Peitz, S.; Wallscheid, O.; Klus, S.; Böcker, J.; Dellnitz, M. Koopman Operator Based Finite-Set Model Predictive Control for Electrical Drives. arXiv 2018, arXiv:1804.00854.

201. Duriez, T.; Brunton, S.L.; Noack, B.R. Machine Learning Control-Taming Nonlinear Dynamics and Turbulence; Springer: Berlin/Heidelberg, Germany, 2017.

(C) 2018 by the authors. Licensee MDPI, Basel, Switzerland. This article is an open access article distributed under the terms and conditions of the Creative Commons Attribution (CC BY) license (http:/ / creativecommons.org/licenses/by/4.0/). 